\title{
Slepian-Wolf Coding for Broadcasting with Cooperative Base-Stations
}

\author{
Roy Timo and Michèle Wigger
}

\begin{abstract}
We propose a base-station (BS) cooperation model for broadcasting a discrete memoryless source in a cellular or heterogeneous network. The model allows the receivers to use helper BSs to improve network performance, and it permits the receivers to have prior side information about the source. We establish the model's information-theoretic limits in two operational modes: In Mode 1, the helper BSs are given information about the channel codeword transmitted by the main BS, and in Mode 2 they are provided correlated side information about the source. Optimal codes for Mode 1 use hash-and-forward coding at the helper BSs; while, in Mode 2, optimal codes use source codes from Wyner's helper source-coding problem at the helper BSs. We prove the optimality of both approaches by way of a new list-decoding generalisation of $[8$, Thm. 6], and, in doing so, show an operational duality between Modes 1 and 2.
\end{abstract}

\section{Introduction \& MAIN ReUlts}

$\mathbf{T}$ HE proliferation of wireless communications devices presents significant performance challenges for cellular networks, and it will require more sophisticated heterogeneous networks in the near future [1, 2]. A powerful methodology for improving performance is centered on the idea of base-station (BS) cooperation: Instead of operating independently, future BSs will coordinate encoding and decoding operations using information shared over backbone networks. The tremendous potential of BS cooperation has been widely investigated [3][5]; however, despite many advances, there remains significant challenges in understanding and exhausting the benefits of cooperation. Indeed, the fundamental limits of cooperation are fully understood in very few settings [4].

To help understand the full potential of BS cooperation, we consider a simple, but rather useful, broadcast model. The setup for two receivers is shown in Figure 1. A source $X$ is to be reliably transmitted over a broadcast channel to many receivers, and the idea is to improve network performance by allowing the receivers to be assisted by helper BSs. In a future heterogenous network, for example, the helpers may be pico or femto BSs operating within the main macro cell on orthogonal channels [6]. Alternatively, the helpers may be WiFi hotspots through which traffic is diverted from a heavily loaded cellular network [7]. The purpose of this paper is to characterise the model's information-theoretic limits, and to provide architectural insights for optimal codes.

R. Timo is an Alexander von Humboldt research fellow with the Technische Universität München, e-mail roy.timo@tum.de.

M. Wigger is with Telecom ParisTech, e-mail michele.wigger@telecomparistech.fr.

This work was supported by the city of Paris under the programme "Emergences" and the Alexander von Humboldt Foundation.

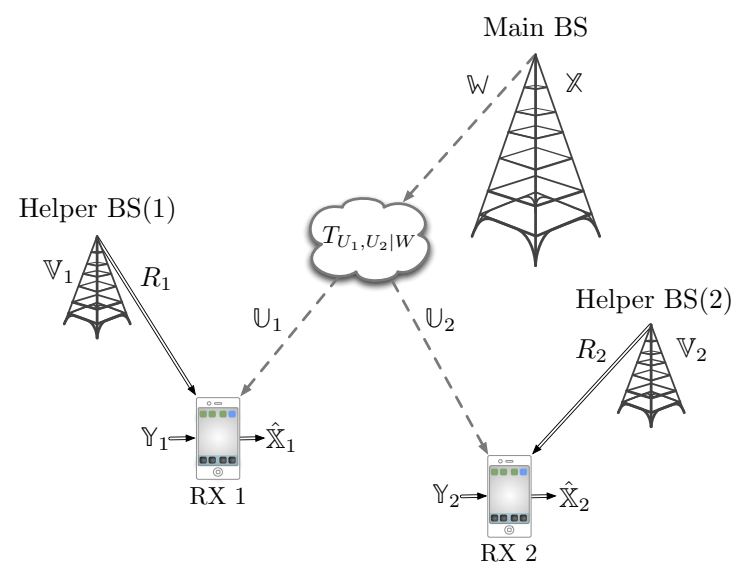

Fig. 1. Broadcasting with helper BSs and receiver side information.

We assume that the broadcast channel from the main BS is discrete and memoryless, and the channels from the helper BSs are noiseless and rate-limited. Although this setup does not capture all modes of cooperation, it nevertheless has enough sophistication to provide insight into some important coding challenges. For example, consider the idea of augmenting traffic flow in a cellular network via a WiFi hotspot: The hotspot's radio-access technology is orthogonal to that of the cellular network, and a cellular network engineer can well approximate the WiFi link by a noiseless rate-limited channel. A natural question is then: What coding techniques at the BSs and WiFi hotspot yield the best overall performance?

Within the above framework, we consider two operational modes.

- Mode 1: The helper BSs are given side information about the channel codeword transmitted by the main BS.

- Mode 2: The helper BSs are given correlated side information about the source $\mathbf{X}$.

We will see that optimal codes for Mode 1 combine virtualbinning from Slepian-Wolf Coding over Broadcast Channels [8] with hash-and-forward coding for the primitive relay channel [9]. Optimal codes for Mode 2, on the other hand, combine virtual binning with source codes from Wyner's helper side-information problem [10]. We prove the optimality of both codes by way of a new list-decoding generalisation of [8, Thm. 6], and, in doing so, show an operational duality between Modes 1 and 2 .

The paper is organised as follows. The BS cooperate model is defined in Section [II and our results are summarised in Section III. We introduce and solve a list-decoding broadcast 
problem in Sections IV through VI. Finally, we prove the BS cooperation results in Sections VII to $\mathrm{X}$

\section{PRELIMINARIES}

\section{A. Notation}

We denote random variables by uppercase letters, e.g. $A$; their alphabets by calligraphic typeface, e.g. $\mathcal{A}$; and elements of an alphabet by lowercase letters, e.g. $a \in \mathcal{A}$. The cartesian product of alphabets $\mathcal{A}$ and $\mathcal{B}$ is $\mathcal{A} \times \mathcal{B}$, and the $n$-fold cartesian product of $\mathcal{A}$ is $\mathcal{A}^{n}$. When $n$ is clear from context, we use boldface notation for a sequence of $n$ random variables on a common alphabet, e.g. $\mathbb{A}=\left(A_{1}, A_{2}, \ldots, A_{n}\right) \in \mathcal{A}^{n}$.

\section{B. Source and Channel Setup}

The main $\mathrm{BS}$ is required to communicate a source

$$
\text { X }=\left(X_{1}, X_{2}, \ldots, X_{n_{\mathrm{s}}}\right)
$$

over a discrete memoryless broadcast channel to $K$ receivers with side information; the side information at receiver $k$, for $k \in\{1,2, \ldots, K\}$, is denoted by

$$
\mho_{k}=\left(Y_{k, 1}, Y_{k, 2}, \ldots, Y_{k, n_{\mathrm{s}}}\right) .
$$

For example, $\mathbb{X}$ and $\bigvee_{k}$ may be the current and previous states of a mobile application, the global and local contents of a cloud storage drive, or the current and previous frames of a video feed. Alternatively, specific choices of $X$ and $\Psi_{k}$ lead to the bi-directional broadcast channel and complementary side information model [11]-[14]. For generality, let us only assume that the source and side information are emitted by a discrete memoryless source ${ }^{1}$. That is,

$$
\left(\mathbb{X}, \mathbb{Y}_{1}, \mathbb{Y}_{2}, \ldots, \mathbb{Y}_{K}\right):=\left\{\left(X_{i}, Y_{1, i}, Y_{2, i}, \ldots, Y_{K, i}\right)\right\}_{i=1}^{n_{\mathrm{s}}}
$$

is a sequence of $n_{\mathrm{s}}$ independent and identically distributed (iid) source/side-information tuples $\left(X, Y_{1}, Y_{2}, \ldots, Y_{K}\right)$ defined by a fixed, but arbitrary, joint probability mass function (pmf) on the Cartesian product space $\mathcal{X} \times \mathcal{Y}_{1} \times \mathcal{Y}_{2} \times \cdots \times \mathcal{Y}_{K}$.

Let $\mathcal{W}$ denote the broadcast channel's input alphabet and $\mathcal{U}_{k}$ its output alphabet at receiver $k$. The main BS transmits

$$
\mathbb{W}:=f(\mathbb{R})
$$

over the broadcast channel, where $f: \mathcal{X}^{n_{\mathrm{s}}} \longrightarrow \mathcal{W}^{n_{\mathrm{c}}}$ is the BS's encoder and $\mathbb{W}=\left(W_{1}, W_{2}, \ldots, W_{n_{\mathrm{c}}}\right)$ is a codeword with $n_{\mathrm{c}}$ symbols. The ratio of channel symbols to source symbols,

$$
\kappa:=\frac{n_{\mathrm{c}}}{n_{\mathrm{s}}},
$$

is called the bandwidth expansion factor.

Receiver $k$ observes $\mathbb{U}_{k}=\left(U_{k, 1}, U_{k, 2}, \ldots, U_{k, n_{\mathrm{c}}}\right)$ from the channel. The channel outputs, across all receivers, conditionally depend on the codeword $\mathbb{W}$ via the memoryless law

$$
\begin{aligned}
\mathbb{P}\left[\mathbb{U}_{1}=\mathbb{U}_{1}, \mathbb{U}_{2}=\mathbb{U}_{2}, \ldots,\right. & \left.\mathbb{U}_{K}=\mathbb{U}_{K} \mid \mathbb{W}=\mathbb{W}\right] \\
= & \prod_{i=1}^{n_{\mathrm{c}}} T\left(u_{1, i}, u_{2, i}, \ldots, u_{K, i} \mid w_{i}\right),
\end{aligned}
$$

where $\mathbb{w} \in \mathcal{W}^{n_{\mathrm{c}}}, u_{k} \in \mathcal{U}_{k}^{n_{\mathrm{c}}}$ and $T\left(u_{1}, \ldots, u_{K} \mid w\right)$ is a fixed, but arbitrary, conditional probability.

\footnotetext{
${ }^{1}$ It is possible to extend this research to discrete ergodic sources using, for example, the methods of [15]. However, discrete memoryless sources lead to more instructive proofs with less technical and notational difficulties.
}

\section{No Base-Station Cooperation}

Momentarily suppose that there is no BS cooperation, and that the source is to be losslessly reconstructed using only the channel outputs and side information at each receiver. In this setting, reliable communication is possible if (and only if) there exists a pmf $P_{W}$ on $\mathcal{W}$ such that [8]

$$
H\left(X \mid Y_{k}\right)<\kappa I\left(W ; U_{k}\right), \quad \forall k,
$$

where $\left(W, U_{1}, U_{2}, \ldots, U_{K}\right) \sim P_{W}(\cdot) T(\cdot \mid \cdot)$. The necessity and sufficiency of (1) for reliable communication is an elegant and powerful result with applications throughout network information theory; for example, consider [11]-[14] and [16][19]. Indeed, a new list-decoding generalisation of (1) will play a central role in this paper.

\section{Base-Station Cooperation}

Let us now return to the BS cooperation model. The helper $\mathrm{BS}$ of receiver $k$, denoted $\mathrm{BS}(k)$, obtains side information

$$
\mathbb{V}_{k}=\left(V_{k, 1}, V_{k, 2}, \ldots, V_{k, n_{\mathrm{h}}}\right)
$$

about the source $\mathbb{X}$ or the codeword $\mathbb{W}$ via a backbone network. Here $n_{\mathrm{h}}=n_{\mathrm{s}}$ (resp. $n_{\mathrm{h}}=n_{\mathrm{c}}$ ) when $\mathrm{BS}(k)$ has side information about $\mathcal{X}$ (resp. $\mathbb{W}$ ), and a precise definition of $\mathbb{V}_{k}$ will be given shortly. $\mathrm{BS}(k)$ sends

$$
M_{k}:=f_{k}\left(\mathbb{V}_{k}\right)
$$

over a noiseless channel to receiver $k$, where $f_{k}: \mathcal{V}^{n_{\mathrm{h}}} \rightarrow\{1,2$, $\left.\ldots,\left\lfloor 2^{n_{\mathrm{s}} R_{k}}\right\rfloor\right\}$ is $\mathrm{BS}(k)$ 's encoder and $R_{k}$ is its rate (in bits per source symbo $3^{3}$. Receiver $k$ attempts to recover the source via

$$
\hat{\mathbb{X}}_{k}:=g_{k}\left(\mathbb{U}_{k}, \mho_{k}, M_{k}\right) \text {, }
$$

where $g_{k}: \mathcal{U}_{k}^{n_{\mathrm{c}}} \times \mathcal{Y}_{k}^{n_{\mathrm{s}}} \times\left\{1,2, \ldots,\left\lfloor 2^{n_{\mathrm{s}} R_{k}}\right\rfloor\right\} \longrightarrow \mathcal{X}^{n_{\mathrm{s}}}$ is the receiver's decoder. The collection of all encoders and decoders is called an $\left(n_{\mathrm{s}}, n_{\mathrm{c}}, R_{1}, R_{2}, \ldots, R_{K}\right)$-code.

\section{E. Mode 1 (helper side information about the codeword $\mathbb{W}$ )}

Suppose that $\mathbb{V}_{k}$ is the entire codeword $\mathbb{W}$ or a scalar quantised version thereof. Quantisation is appropriate, for example, when the backbone network is rate limited. More formally, let $\phi_{k}: \mathcal{W} \rightarrow \mathcal{V}_{k}$ be an arbitrary but given deterministic mapping (scalar quantiser) and

$$
V_{k, i}:=\phi_{k}\left(W_{i}\right), \quad \forall i .
$$

The main problem of interest is to determine when reliable communication is achievable in the following sense.

Definition 1: Fix the bandwidth expansion factor $\kappa$, helper BS rates $\boldsymbol{R}:=\left(R_{1}, R_{2}, \ldots, R_{K}\right)$, and scalar quantisers $\phi:=$ $\left(\phi_{1}, \phi_{2}, \ldots, \phi_{K}\right)$. We say that a source/side information tuple $\left(X, Y_{1}, Y_{2}, \ldots, Y_{K}\right)$ is $(\kappa, \boldsymbol{R}, \boldsymbol{\phi})$-achievable if for any $\epsilon>0$ there exists an $\left(n_{\mathrm{s}}, n_{\mathrm{c}}, R_{1}, R_{2}, \ldots, R_{K}\right)$-code such that

$$
\frac{n_{\mathrm{c}}}{n_{\mathrm{s}}}=\kappa \quad \text { and } \quad \mathbb{P}\left[\hat{\mathbb{K}}_{k} \neq \mathrm{X}\right] \leq \epsilon, \quad \forall k,
$$

holds for sufficiently large $n_{\mathrm{s}}$ and $n_{\mathrm{c}}$.

\footnotetext{
${ }^{2}$ Replace the strict inequality in (1) with an inequality.

${ }^{3}$ Here we have synchronised the rate $R_{k}$ to the number of source symbols $n_{\mathrm{s}}$. Alternatively, one could synchronise $R_{k}$ to the number of channel symbols
} by replacing $n_{\mathrm{s}}$ with $n_{\mathrm{c}}$ in the definition of $f_{k}$. 


\section{F. Mode 2 (helper side information about the source $\mathbb{X}$ )}

Suppose that $\mathbb{V}_{k}$ is directly correlated with the source and side information. That is, assume $\left(\mathbb{X}, \mathbb{V}_{1}, \mathbb{Y}_{2}, \ldots, \mathbb{Y}_{K}, \mathbb{V}_{1}, \mathbb{V}_{2}\right.$, $\left.\ldots, \mathbb{V}_{K}\right)$ is emitted by an arbitrary discrete memoryless source and thus is a sequence of $n_{\mathrm{s}}$ iid tuples $\left(X, Y_{1}, Y_{2}, \ldots, Y_{K}, V_{1}\right.$, $\left.V_{2}, \ldots, V_{K}\right)$. We are interested in the following definition of achievability.

Definition 2: Fix the bandwidth expansion factor $\kappa$ and helper $\mathrm{BS}$ rates $\boldsymbol{R}:=\left(R_{1}, R_{2}, \ldots, R_{K}\right)$. We say that a source/side information tuple $\left(X, Y_{1}, Y_{2}, \ldots, Y_{K}, V_{1}, V_{2}, \ldots\right.$, $\left.V_{K}\right)$ is $(\kappa, \boldsymbol{R})$-achievable if for any $\epsilon>0$ there exists an $\left(n_{\mathrm{s}}\right.$, $\left.n_{\mathrm{c}}, R_{1}, R_{2}, \ldots, R_{K}\right)$-code such that (2) holds for sufficiently large $n_{\mathrm{s}}$ and $n_{\mathrm{c}}$.

\section{INFORMATION-THEORETIC LIMITS OF BS COOPERATION}

We now give necessary and sufficient conditions for a source/side-information tuple to be achievable in the sense of Definitions 1 and 2. We then present results for some simple variations of the BS cooperation model, and we conclude the section with a discussion of the existing literature.

\section{A. Mode 1}

Theorem 1: Fix the helper BS rates $\boldsymbol{R}$, bandwidth expansion factor $\kappa$ and quantisers $\phi$. A source/side-information tuple $\left(X, Y_{1}, Y_{2}, \ldots, Y_{K}\right)$ is $(\kappa, \boldsymbol{R}, \phi)$-achievable if (and only if) ${ }^{4}$ there exists a pmf $P_{W}$ on $\mathcal{W}$ such that for all $k$

$$
H\left(X \mid Y_{k}\right) \stackrel{*}{<} \kappa I\left(W ; U_{k}\right)+\min \left\{R_{k}, \kappa I\left(W ; V_{k} \mid U_{k}\right)\right\},
$$

where $\left(W, U_{1}, U_{2}, \ldots, U_{K}\right) \sim P_{W}(\cdot) T(\cdot \mid \cdot)$ and $V_{k}=\phi_{k}(W)$.

Theorem 1 is proved in Sections VII and VIII

\section{B. Mode 2}

Theorem 2: Fix the helper BS rates $\boldsymbol{R}$ and bandwidth expansion factor $\kappa$. A source/side-information tuple $\left(X, Y_{1}, Y_{2}\right.$, $\left.\ldots, Y_{K}, V_{1}, V_{2}, \ldots, V_{K}\right)$ is $(\kappa, \boldsymbol{R})$-achievable if (and only if) ${ }^{4}$ there exists a pmf $P_{W}$ on $\mathcal{W}$ and $K$ auxiliary random variables $\left(A_{1}, A_{2}, \ldots, A_{K}\right)$ such that for all $k$ we have the Markov chain $\left(X, Y_{k}\right) \leftrightarrow V_{k} \leftrightarrow A_{k}$,

$$
R_{k} \stackrel{*}{>} I\left(V_{k} ; A_{k} \mid Y_{k}\right)
$$

and

$$
H\left(X \mid A_{k}, Y_{k}\right) \stackrel{*}{<} \kappa I\left(W ; U_{k}\right),
$$

where $\left(W, U_{1}, U_{2}, \ldots, U_{K}\right) \sim P_{W}(\cdot) T(\cdot \mid \cdot)$.

Theorem 2 is proved in Sections IX and $\mathrm{X}$.

Remark 1: When computing Theorem 2, we can assume that the alphabet of $A_{k}$ has a cardinality of at most $\left|\mathcal{V}_{k}\right|$.

\footnotetext{
${ }^{4}$ For the "only if" direction replace the strict inequality $(*)$ with an inequality.
}

\section{Example for Theorem 2}

Consider Theorem 2, and choose $\left(\rho_{1}, \ldots, \rho_{K}\right) \in[0,1 / 2]^{K}$. Suppose that the source is uniform and binary, $X \sim \operatorname{Bern}(1 / 2)$; there is no receiver side information, $Y_{k}=$ constant; and define helper $\mathrm{BS}(k)$ 's side information to be

$$
\left.V_{k}:=X \oplus Z_{k}, \quad \text { (modulo } 2\right),
$$

where $Z_{k}:=\operatorname{Bern}\left(\rho_{k}\right)$ is independent additive binary noise. The source / side-information tuple $\left(X, V_{1}, V_{2}, \ldots, V_{K}\right)$ is achievable if (and only if) ${ }^{4}$ there exists a pmf $P_{W}$ on $\mathcal{W}$ and $\left(\alpha_{1}, \alpha_{2}, \ldots, \alpha_{K}\right) \in[0,1 / 2]^{K}$ such that for all $k$ we have

$$
R_{k} \stackrel{*}{>} 1-h\left(\alpha_{k}\right) \text { and } h\left(\alpha_{k} \star \rho_{k}\right) \stackrel{*}{<} \kappa I\left(W ; U_{k}\right),
$$

where

$h(a):= \begin{cases}-a \log _{2} a-(1-a) \log _{2}(1-a), & a \in(0,1 / 2], \\ 0, & a=0 .\end{cases}$

is the binary entropy function and

$$
a \star b:=a(1-b)+(1-a) b, \quad 0 \leq a, b \leq 1 .
$$

The above example is an application of Wyner's binary helper source coding problem [10] (see also [35]-[37] and [29. Thm. 10.2]). To see why (6) holds, consider the following: Let $\left(A_{1}, A_{2}, \ldots, A_{K}\right)$ be any tuple of auxiliary random variables satisfying the conditions of Theorem 2 . We first notice that

$$
\begin{aligned}
H\left(X \mid V_{k}\right) \stackrel{\mathrm{a}}{=} & h\left(\rho_{k}\right) \\
& \stackrel{\mathrm{b}}{\leq} H\left(X \mid A_{k}\right) \stackrel{\mathrm{c}}{=} H\left(X \mid A_{k}, Y_{k}\right) \stackrel{\mathrm{d}}{\leq} 1, \quad \forall k,
\end{aligned}
$$

where step (a) follows from (5); (b) notes that $X \leftrightarrow V_{k} \leftrightarrow A_{k}$ forms a Markov chain and applies the data processing lemma; (c) follows because $Y_{k}$ is a constant; and (d) follows because $X$ is binary. From (7), it follows that we can find $\alpha_{k} \in[0,1 / 2]$, for all $k$, such that

$$
H\left(X \mid A_{k}, Y_{k}\right)=h\left(\alpha_{k} \star \rho_{k}\right) .
$$

In addition, we have

$$
\begin{aligned}
I\left(V_{k} ; A_{k} \mid Y_{k}\right) \stackrel{\mathrm{a}}{=} I\left(V_{k} ; A_{k}\right) & \\
& \stackrel{\mathrm{b}}{=} 1-H\left(V_{k} \mid A_{k}\right) \stackrel{\mathrm{c}}{\geq} 1-h\left(\alpha_{k}\right) .
\end{aligned}
$$

Here (a) follows because $Y_{k}$ is a constant, and (b) follows because $V_{k} \sim \operatorname{Bern}(1 / 2)$ and thus $H\left(V_{k}\right)=1$. Step (c) invokes Mrs Gerber's Lemma [35, 36] (see also [29, p. 19]) to upper bound $H\left(V_{k} \mid A_{k}\right)$ by $h\left(\alpha_{k}\right)$. More specifically, we have $X=V_{k} \oplus Z_{k}$ and $Z_{k} \sim \operatorname{Bern}\left(\rho_{k}\right)$. Since $X \leftrightarrow V_{k} \leftrightarrow A_{k}$, it follows that

$$
\begin{aligned}
& 0=I\left(A_{k} ; V_{k} \oplus Z_{k} \mid V_{k}\right)=I\left(A_{k} ; Z_{k} \mid V_{k}\right) \\
& \Rightarrow A_{k} \leftrightarrow V_{k} \leftrightarrow Z_{k} .
\end{aligned}
$$

Combining (9) with $I\left(V_{k} ; Z_{k}\right)=0$ shows that $Z_{k}$ is independent of $\left(V_{k}, A_{k}\right)$ and hence Mrs Gerber's Lemma applies.

The above discussion shows that (8) holds for any choice of auxiliary random variables satisfying the conditions of Theorem 2. To complete the example, we need only find auxiliary random variables for which $(8)$ holds with equality. To this end, simply let $A_{k}$ be the output of a binary symmetric channel with input $V_{k}$ and crossover probability $\alpha_{k}$. 


\section{Mixed modes}

Suppose that some helper BSs have information about the codeword $\mathbb{W}$, while others have information about the source $\mathcal{X}$ - a mix of Modes 1 and 2. Let $\mathcal{K}_{1}$ and $\mathcal{K}_{2}$ denote the index sets of Mode 1 and 2 helper BSs respectively. It can be argued from Theorems 1 and 2 that a source/side-information tuple is achievable if (and only if) ${ }^{4}$ there exists a pmf $P_{W}$ on $\mathcal{W}$ and $\left|\mathcal{K}_{2}\right|$ auxiliary random variables $\left\{A_{k} ; k \in \mathcal{K}_{2}\right\}$ such that $A_{k} \leftrightarrow V_{k} \leftrightarrow\left(X, Y_{k}\right)$ forms a Markov chain,

$H\left(X \mid Y_{k}\right) \stackrel{*}{<} \kappa I\left(W ; U_{k}\right)+\min \left\{R_{k}, \kappa I\left(W ; V_{k} \mid U_{k}\right)\right\}, \forall k \in \mathcal{K}_{1}$, and

$$
\begin{aligned}
& R_{k}{\stackrel{*}{>} I\left(V_{k} ; A_{k} \mid Y_{k}\right) \quad \text { and }}^{H\left(X \mid A_{k}, Y_{k}\right) \stackrel{*}{<} \kappa I\left(W ; U_{k}\right), \quad \forall k \in \mathcal{K}_{2} .}
\end{aligned}
$$

\section{E. Broadcast capacity with helpers}

Consider Mode 1 for the bandwidth-matched case $n_{\mathrm{s}}=$ $n_{\mathrm{c}}=n$, and fix a positive rate $R^{*}$. Suppose that there is no side information and the main $\mathrm{BS}$ is required to broadcast a discrete rate $R^{*}$ message $M$ to the receivers, where $M$ is uniformly distributed on $\left\{1,2, \ldots,\left\lfloor 2^{n R^{*}}\right\rfloor\right\}$. For example, in Theorem 1 suppose that $2^{R^{*}}$ is an integer, $\kappa=1, Y_{k}=$ constant and $M=\mathbb{X}$, where $\mathbb{X}$ is iid with a uniform distribution on $\{1,2$, $\left.\ldots, 2^{R^{*}}\right\}$. Then $H\left(X \mid Y_{k}\right)=H(X)=R^{*}$ for all $k$.

Given helper rates $\boldsymbol{R}$, we can define the helper capacity $C(\boldsymbol{R})$ to be the supremum of all achievable message rates $R^{*}$; that is, those rates $R^{*}$ for which there exists a sequence of codes with vanishing probability of decoding error. It can be argued from Theorem 1 that

$$
\begin{aligned}
C(\boldsymbol{R})=\max _{P_{W}} \min _{k}[I(W & \left.; U_{k}\right) \\
& \left.+\min \left\{R_{k}, I\left(W ; V_{k} \mid U_{k}\right)\right\}\right],
\end{aligned}
$$

where the maximisation is taken over all pmfs $P_{W}$ on $\mathcal{W}$.

If the channel outputs are defined over a common alphabet, say $\mathcal{U}_{k}=\mathcal{U}$ for all $k$, then $(10)$ is a type of compound channel capacity with relays. Indeed, one recovers the compound channel capacity theorem [20, 21] upon setting $R_{k}=0$ in $[10$.

\section{F. Bidirectional broadcast channel with helpers}

Consider Mode 1 with two receivers for the bandwidth matched case $n_{\mathrm{s}}=n_{\mathrm{c}}=n$, and fix positive rates $R_{1}^{*}$ and $R_{2}^{*}$. Recall the bidirectional setup of [11]: The main BS has two independent uniformly distributed messages $M_{1}$ and $M_{2}$ on $\left\{1,2, \ldots,\left\lfloor 2^{n R_{1}^{*}}\right\rfloor\right\}$ and $\left\{1,2, \ldots,\left\lfloor 2^{n R_{2}^{*}}\right\rfloor\right\}$ respectively; receiver 1 has $M_{1}$ as side information and requires $M_{2}$; and receiver 2 has $M_{2}$ as side information and requires $M_{1}$. For example, in Theorem 1 suppose that $\kappa=1,2^{R_{1}^{*}}$ and $2^{R_{2}^{*}}$ are integers, $M_{1}=\mathbb{X}_{1}=\mathbb{Y}_{1}$ and $M_{2}=\mathbb{X}_{2}=\mathbb{Y}_{2}$, where $X_{1}$ and $X_{2}$ are independent with iid uniform distributions on $\left\{1,2, \ldots, 2^{R_{1}^{*}}\right\}$ and $\left\{1,2, \ldots, 2^{R_{2}^{*}}\right\}$ respectively. Then, setting $\mathbb{X}=\left(\mathfrak{X}_{1}, \mathfrak{X}_{2}\right)$ gives

$$
\begin{aligned}
& H\left(X \mid Y_{1}\right)=H\left(X_{1}, X_{2} \mid X_{1}\right)=H\left(X_{2}\right)=R_{2}^{*} \quad \text { and } \\
& H\left(X \mid Y_{2}\right)=H\left(X_{1}, X_{2} \mid X_{2}\right)=H\left(X_{1}\right)=R_{1}^{*} .
\end{aligned}
$$

For fixed helper rates $\left(R_{1}, R_{2}\right)$, we can define the helper capacity region $\mathcal{C}\left(R_{1}, R_{2}\right)$ to be closure of the set of all $\left(R_{1}, R_{2}\right)$-achievable rate pairs $\left(R_{1}^{*}, R_{2}^{*}\right)$. It can be argued from Theorem 1 that $\mathcal{C}\left(R_{1}, R_{2}\right)$ is equal to the set of all $\left(R_{1}^{*}, R_{2}^{*}\right)$ for which there exists a pmf $P_{W}$ on $\mathcal{W}$ such that

$$
\begin{aligned}
& R_{1}^{*} \leq I\left(W ; U_{2}\right)+\min \left\{R_{2}, I\left(W ; V_{2} \mid U_{2}\right)\right\} \\
& R_{2}^{*} \leq I\left(W ; U_{1}\right)+\min \left\{R_{1}, I\left(W ; V_{1} \mid U_{1}\right)\right\} .
\end{aligned}
$$

\section{G. Other work \& Operational source-channel separation}

Consider Mode 1 and Theorem 1, If the helper rates are all set to zero, then (3) becomes

$$
H\left(X \mid Y_{k}\right) \stackrel{*}{<} \kappa I\left(W ; U_{k}\right), \quad \forall k,
$$

and we recover the setup of (1). Now suppose that for a given pmf $P_{W}$ and scalar quantisers $\phi$ we have $R_{k}>\kappa H\left(V_{k} \mid U_{k}\right)$ for all $k$. If $\left(\mathbb{V}_{k}, \mathbb{U}_{k}\right)$ behaves like a discrete memoryless source, then $\mathrm{BS}(k)$ can reliably send $\mathbb{V}_{k}$ to receiver $k$ using a Slepian-Wolf code of rate $R_{k}$ [22]. The receiver effectively has the combined channel output $\left(\mathbb{U}_{k}, \mathbb{V}_{k}\right)$. Since (3) simplifies to

$$
H\left(X \mid Y_{k}\right) \stackrel{*}{<} \kappa I\left(W ; U_{k}, V_{k}\right), \quad \forall k,
$$

we again return to the result in (1), where the $k$-th channel output $U_{k}$ is replaced by $\left(U_{k}, V_{k}\right)$.

For other helper rates, we note the similarity of (3) to Kim's capacity theorem [9, Thm. 1] for the primitive relay channel. Intuitively, the right hand side of (3) is the maximum rate at which information can be sent to receiver $k$. This intuition, however, should treated with care because, for example, the classical Shannon approach of strictly separating source and channel coding is suboptima 5 Nonetheless, it is natural to wonder whether Kim's simple timesharing proof of [9, Thm. 1] can be modified to prove Theorem 1. While we do not take the timesharing approach in this paper, D. Gündüz has noticed that it may indeed be possible to give such a proof of Theorem 1 using the semiregular encoding and backward decoding techniques developed in [19, App. B] (these techniques, for example, give an alternative proof of the no-cooperation case shown in (1)).

The single-letter characterisations in Theorems 1 and 2 depend only on the marginal source and channel distributions, instead of the complete joint source-channel distribution ${ }^{6}$ the latter being more typical in the joint source-channel coding literature, e.g., see [38]. The separation of source and channel variables in Theorems 1 and 2 is reminiscent of operational separation described in $[8]$ and can be similarly understood 7 Indeed, in both modes we will see that it is optimal to separate the source, channel and helper codebooks as well as the encoders, but joint decoding across all three codebooks is required. In particular, the approach taken in this paper is to first require that receiver $k$ decodes a list of likely source sequences

\footnotetext{
${ }^{5}$ To see why strict source-channel separation fails, set $R_{k}=0$ for all $k$ and consider the examples in [8].

${ }^{6}$ All of the entropy and mutual information functions in Theorems 1 and 2 depend on either the source variables or the channel variables, but not both.

${ }^{7}$ More detailed discussions on the various types of source-channel separation can be found in \begin{tabular}{ll|l|l|}
8 & 16 & $19]$
\end{tabular}
} 
using a joint source-channel decoder on its channel output $\mathbb{U}_{k}$ and side information $\mathbb{Y}_{k}$. The receiver then determines the correct source sequence, within this list, using the helper BS's message and codebook. The list decoding approach is particularly useful because it highlights an operation duality between Modes 1 and 2: The helper BS's task in both modes is to help the receiver resolve the correct source sequence from the receiver's list.

The problem setup of Mode 2 is a special case of the more general problem considered by Gündüz, Erkip, Goldsmith and Poor in [19, Sec. V]. Gündüz et al. presented an achievability result for the general problem in [19. Thm. 3], however, this result is not optimal in the case of Theorem 2. In this paper, helper $\mathrm{BS}(k)$ provides information directly about the source $\mathbb{X}$ via a 'quantised' version of $\mathbb{V}_{k}$. The quantisation is specified by the auxiliary random variable $A_{k}$ in a similar way to the quantisation in Wyner's helper side-information problem [10], [23, p. 575] or the Wyner-Ziv rate-distortion problem [24].

Comparing Theorems 1 and 2. Increasing the helper rates in Theorem 2 allows larger 'quantisation rates' and reductions in the left hand side of (4b). In contrast, increasing the helper rates in Theorem 1 improves the 'relay capacity' and increases the right hand side of (3). We will see that these properties are dual consequences of the same random-coding idea.

Finally, we note that Theorems 1 and 2 are existential statements that do not give constructive arguments for lowcomplexity codes. That being said, however, the single-letter expressions and (as we will see) the structure of the randomcoding achievability proofs give insight into the architecture of good low-complexity codes. For example, the hash-andforward technique used in Mode 1 is similar to distributed source coding using LDPC codes [25]. Similarly, in Mode 2, preliminary work suggests that (nonlinear) trellis codes and rate-distortion codes perform well for quantising $\mathbb{V}_{k}$ [26]. Finally, recent work [39] suggests that repeat-accumulate codes can be useful for Slepian-Wolf coding over broadcast channels.

\section{Slepian-Wolf Coding over Broadcast CHANNELS WITH LIST DECODING}

It is useful to consider a list-decoding extension to (1) before proving Theorems 1 and 2. In this section, suppose that there is no $\mathrm{BS}$ cooperation and the receivers employ list decoding.

\section{A. Setup and Main Result}

Let $\Omega(L):=\left\{\mathcal{L} \subseteq \mathcal{X}^{n_{\mathrm{s}}}:|\mathcal{L}|=L\right\}$ denote the collection of all subsets of $\mathcal{X}^{n_{\mathrm{s}}}$ with cardinality $L$. An $\left(n_{\mathrm{s}}, n_{\mathrm{c}}, L_{1}, L_{2}, \ldots\right.$, $\left.L_{K}\right)$ list code is a collection of $(K+1)$ maps $\left(f, g_{1}, g_{2}\right.$, $\left.\ldots, g_{K}\right)$, where

$$
f: \mathcal{X}^{n_{\mathrm{s}}} \longrightarrow \mathcal{W}^{n_{\mathrm{c}}}
$$

is the encoder at the transmitter and

$$
g_{k}: \mathcal{U}_{k}^{n_{\mathrm{c}}} \times \mathcal{Y}_{k}^{n_{\mathrm{s}}} \longrightarrow \Omega\left(L_{k}\right)
$$

is the list decoder at receiver $k$. Upon observing the channel output $\mathbb{U}_{k}$ and side information $\mathbb{Y}_{k}$, receiver $k$ computes the list

$$
\mathcal{L}_{k}:=g_{k}\left(\mathbb{U}_{k}, \mathbb{Y}_{k}\right)
$$

An error is declared at receiver $k$ if $\mathfrak{X} \notin \mathcal{L}_{k}$.

If (1) holds, then [8, Thm. 6] guarantees the existence of a sequence of list codes with $\left|\mathcal{L}_{k}\right|=1$ and $\mathbb{P}\left[\mathcal{X} \notin \mathcal{L}_{k}\right] \rightarrow 0$ for all $k$. On the other hand: If (1) does not hold, then $\left|\mathcal{L}_{k}\right|$ must grow exponentially in $n_{\mathrm{s}}$ to ensure $\mathbb{P}\left[\mathbb{K} \notin \mathcal{L}_{k}\right] \rightarrow 0$. We are concerned with the smallest such exponent.

Definition 3: Fix the bandwidth expansion factor $\kappa$ and list exponents $\boldsymbol{D}=\left(D_{1}, D_{2}, \ldots, D_{K}\right)$, with $D_{k} \geq 0, \forall k$. We say that the pair $(\kappa, D)$ is achievable if for any $\epsilon>0$ there exists a $\left(n_{\mathrm{s}}, n_{\mathrm{c}}, L_{1}, L_{2}, \ldots, L_{K}\right)$ list code such that

$$
\begin{gathered}
\frac{n_{\mathrm{c}}}{n_{\mathrm{s}}}=\kappa, \\
L_{k} \leq 2^{n_{\mathrm{s}} D_{k}} \quad \text { and } \quad \mathbb{P}\left[\mathbb{X} \notin \mathcal{L}_{k}\right] \leq \epsilon, \forall k,
\end{gathered}
$$

where $n_{\mathrm{s}}$ and $n_{\mathrm{c}}$ are sufficiently large.

The next lemma is proved in Sections $\mathrm{V}$ and $\mathrm{VI}$

Lemma 3: $(\kappa, \boldsymbol{D})$ is achievable if (and only if ${ }^{8}$ there exists a pmf $P_{W}$ on $\mathcal{W}$ such that

$$
D_{k} \stackrel{*}{>} \max \left\{H\left(X \mid Y_{k}\right)-\kappa I\left(W ; U_{k}\right), 0\right\}, \quad \forall k,
$$

where $\left(W, U_{1}, U_{2}, \ldots, U_{K}\right) \sim P_{W}(\cdot) T(\cdot \mid \cdot)$.

Lemma 3 is quite intuitive: The best exponent of receiver $k$ 's list size can be larger, but not smaller, than the equivocation in $X$ given $Y_{k}$ minus the information conveyed over the channel.

Remark 2: Definition 3 is a lossy generalisation of the setup for (11). The standard (per-letter / average distortion) generalisation of (1) is called "Wyner-Ziv Coding over broadcast channels" [17], and it is a formidable open problem that includes Heegard and Berger's rate-distortion function [14, 27, 28] as well as the broadcast capacity region [29].

Remark 3: Definition 3 and Lemma 3 are related to Chia's recent list-decoding result [30, Prop. 1] for Heegard and Berger's rate-distortion problem [27]. For example, suppose that $\kappa=1$ and we replace the memoryless $\mathrm{BC} T(\cdot \mid \cdot)$ in our model with a noiseless source-coding 'index' channel, with alphabet $\left\{1,2, \ldots,\left\lfloor 2^{n_{\mathrm{s}} R_{\mathrm{s}}}\right\rfloor\right\}$. In this case, the mutual information $I\left(W ; U_{k}\right)$ transforms to the source-coding rate $R_{\mathrm{s}}$ and Lemma 3 reduces to [30. Prop. 1]

$$
R_{\mathrm{s}} \stackrel{*}{>} \max _{k}\left\{H\left(X \mid Y_{k}\right)-D_{k}\right\} .
$$

Remark 4: Lemma 3 is consistent with Tuncel's result for unique decoding (1) in the following sense. Suppose that we are interested in unique decoding and hence the all-zero list exponent vector $\boldsymbol{D}=(0,0, \ldots, 0)$. The reverse (converse) assertion of Lemma 3 shows that $(\kappa, \boldsymbol{D})$ is achievable only if

$$
H\left(X \mid Y_{k}\right) \leq \kappa I\left(W ; U_{k}\right), \quad \forall k .
$$

The forward (achievability) assertion of Lemma 3, unfortunately, does not include the all-zero list exponent. It does, however, say the following: Any arbitrarily small positive list exponent $D$ is achievable if (13) holds.

Remark 5: It is natural to combine and extend (1) and Lemma 3 as follows: Suppose that a subset

$$
\mathcal{K}_{\text {List }} \subseteq\{1,2, \ldots, K\}
$$

\footnotetext{
${ }^{8}$ Replace the strict inequality $(*)$ with an inequality.
} 
of receivers employ list decoding as in $12 \mathrm{~b}$, and the remaining receivers

$$
\mathcal{K}_{\text {Unique }}:=\{1,2, \ldots, K\} \backslash \mathcal{K}_{\text {List }}
$$

employ unique decoding as in $\mathbb{P}\left[\hat{\mathcal{K}}_{k} \neq \mathbb{X}\right] \leq \epsilon$ (for example, see [8]). The bandwidth expansion factor $\kappa$ is jointly achievable with list exponents $\left\{D_{k} \geq 0 ; k \in \mathcal{K}_{\text {List }}\right\}$ for the receivers in $\mathcal{K}_{\text {List }}$ and unique decoding for receivers in $\mathcal{K}_{\text {Unique }}$ if (and only if $)^{4}$ there exists a pmf $P_{W}$ on $\mathcal{W}$ such that

$$
D_{k} \stackrel{*}{>} \max \left\{H\left(X \mid Y_{k}\right)-\kappa I\left(W ; U_{k}\right), 0\right\}, \forall k \in \mathcal{K}_{\text {List }}
$$

and

$$
H\left(X \mid Y_{k}\right) \stackrel{*}{<} \kappa I\left(W ; U_{k}\right), \quad \forall k \in \mathcal{K}_{\text {Unique }},
$$

where $\left(W, U_{1}, U_{2}, \ldots, U_{K}\right) \sim P_{W}(\cdot) T(\cdot \cdot \cdot)$. The reverse (converse) assertion of (14) automatically follows from Lemma 3 upon setting $D_{k}=0$ for all $k \in \mathcal{K}_{\text {Unique }}$. As to the forward (achievability) assertion: The random codebook used in 88 . Thm. 6] has the same structure as that used to prove Lemma 3 so we need only combine the error analysis in Section VI with the analysis in [8, Sec. IV] using, say, the union bound. We omit the details.

\section{B. Discussion: List Decoding and the Operational Duality of Theorems 1 and 2}

It turns out that the following approach to BS cooperation is optimal in both modes: Use a good list code on the broadcast channel, and task $\mathrm{BS}(k)$ with helping receiver $k$ determine which element of its decoded list $\mathcal{L}_{k}$ is equal to the source $\mathbb{X}$. This list will, with high probability, include $\mathbb{X}$ and have $\left|\mathcal{L}_{k}\right| \approx 2^{n_{\mathrm{s}} D_{k}}$ elements. To resolve receiver $k$ 's uncertainty, $\mathrm{BS}(k)$ needs to encode its side information $\mathbb{V}_{k}$ at a rate $R_{k}$ that is proportional to the list exponent $D_{k}$. In both modes, the smallest achievable rate $R_{k}$ is fundamentally determined by Lemma 3 . Theorems 1 and 2 are duals in the operational sense that changing from Mode 1 to Mode 2 (or, vice versa) does not change the underlying coding problem - it only changes $\mathrm{BS}(k)$ 's approach to the problem. The side information $\mathbb{V}_{k}$ in Mode 2 is directly correlated with the source $\mathcal{X}$, and, in this setting, it is optimal for $\mathrm{BS}(k)$ to use a good source code from Wyner's 'helper' source coding problem [10]. In Mode 1 , on the other hand, the side information $\mathbb{V}_{k}$ is a scalar quantised version of the channel codeword, and it is optimal for BS $(k)$ to use a version of Kim's 'random-hashing' for the relay channel [9]. The remainder of the paper is devoted to proving Lemma 3 and Theorems 1 and 2

\section{Proof of Lemma 3 - Converse}

Fix $\epsilon>0$, and suppose that we have a $\left(n_{\mathrm{s}}, n_{\mathrm{c}}, L_{1}, L_{2}\right.$, $\left.\ldots, L_{K}\right)$ list code such that 12 holds. As before, let $\mathbb{W}=$ $f(\mathbb{X})$ and $\mathbb{U}_{k}=\left(U_{k, 1}, U_{k, 2}, \ldots, U_{k, n_{\mathrm{c}}}\right)$ denote the transmitted codeword and the channel output at receiver $k$.

The first step mirrors that of [8, Thm. 6]. Consider the $j$-th symbol $W_{j}$ of $\mathbb{W}=\left(W_{1}, W_{2}, \ldots, W_{n_{\mathrm{c}}}\right)$, and let $P_{W_{j}}$ denote its pmf. Construct a timeshared $\operatorname{pmf} P_{\tilde{W}}$ on $\mathcal{W}$ by setting

$$
P_{\tilde{W}}(w):=\frac{1}{n_{\mathrm{c}}} \sum_{j=1}^{n_{\mathrm{c}}} P_{W_{j}}(w), \quad w \in \mathcal{W} .
$$

Let $\left(\tilde{W}, \tilde{U}_{1}, \tilde{U}_{2}, \ldots, \tilde{U}_{K}\right) \sim P_{\tilde{W}}(\cdot) T(\cdot \mid \cdot)$. We have

$$
\begin{aligned}
n_{\mathrm{c}} I\left(\tilde{W} ; \tilde{U}_{k}\right) & \stackrel{\mathrm{a}}{\geq} \sum_{i=1}^{n_{\mathrm{c}}} I\left(W_{i} ; U_{k, i}\right) \\
& \stackrel{\mathrm{b}}{\geq} I\left(\mathbb{W} ; \mathbb{U}_{k}\right) \\
& \stackrel{\mathrm{c}}{\geq} I\left(\mathbb{X} ; \mathbb{U}_{k} \mid \mathbb{Y}_{k}\right) \\
& \stackrel{\mathrm{d}}{=} n_{\mathrm{s}} H\left(X \mid Y_{k}\right)-H\left(\mathbb{K} \mid \mathbb{Y}_{k}, \mathbb{U}_{k}\right) .
\end{aligned}
$$

Notes: (a) use Jensen's inequality [23, Thm. 2.7.4]; (b)

$$
\begin{aligned}
& \sum_{i=1}^{n_{\mathrm{c}}} I\left(W_{i} ; U_{k, i}\right) \\
& \quad=\sum_{i=1}^{n_{\mathrm{c}}}\left(H\left(U_{k, i}\right)-H\left(U_{k, i} \mid W_{i}\right)\right) \\
& \quad \geq H\left(\mathbb{U}_{k}\right)-\sum_{i=1}^{n_{\mathrm{c}}} H\left(U_{k, i} \mid W_{i}\right) \\
& \quad * H\left(\mathbb{U}_{k}\right)-\sum_{i=1}^{n_{\mathrm{c}}} H\left(U_{k, i} \mid \mathbb{W}, U_{k, 1}, U_{k, 2}, \ldots, U_{k, i-1}\right) \\
& \quad=H\left(\mathbb{U}_{k}\right)-H\left(\mathbb{U}_{k} \mid \mathbb{W}\right),
\end{aligned}
$$

where $(*)$ follows because the broadcast channel is memoryless and therefore $U_{k, i} \leftrightarrow W_{i} \leftrightarrow\left(\mathbb{W}, U_{k, 1}, U_{k, 2}, \ldots, U_{k, i-1}\right)$ forms a Markov chain; (c) $\left(\mathbb{X}, \mathbb{V}_{k}\right) \leftrightarrow \mathbb{W} \leftrightarrow \mathbb{U}_{k}$ forms a Markov chain; and (d) the source is iid.

We now use a list-decoding version of Fano's inequality, e.g., see [30, Lem. 1] or [31, Lem. 1]:

$$
\begin{aligned}
H\left(\mathbb{X} \mid \mathbb{Y}_{k}, \mathbb{U}_{k}\right) & \leq \log \left|\mathcal{L}_{k}\right|+1 \\
& +\left(n_{\mathrm{s}} \log |\mathcal{X}|-\log \left|\mathcal{L}_{k}\right|\right) \mathbb{P}\left[\mathfrak{X} \notin \bigcap_{k=1}^{K} \mathcal{L}_{k}\right] .
\end{aligned}
$$

By $(\overline{12 b})$, and since for any $k, \mathbb{P}\left[\mathcal{K} \notin \mathcal{L}_{k}\right] \geq \mathbb{P}\left[\mathcal{K} \notin \cap_{k^{\prime}} \mathcal{L}_{k^{\prime}}\right]$, this inequality implies

$$
H\left(\mathbb{X} \mid \mathbb{Y}_{k}, \mathbb{U}_{k}\right) \leq n_{\mathrm{s}}\left(D_{k}+\varepsilon\left(n_{\mathrm{s}}, \epsilon\right)\right),
$$

where

$$
\varepsilon\left(n_{\mathrm{s}}, \epsilon\right):=\frac{1}{n_{\mathrm{s}}}+\epsilon\left(1+\log |\mathcal{X}|-D_{k}-\epsilon\right) .
$$

Combining (12a), 16) and (17), we have

$$
\kappa I\left(\tilde{W} ; U_{k}\right) \geq H\left(X \mid Y_{k}\right)-D_{k}-\varepsilon\left(n_{\mathrm{s}}, \epsilon\right) .
$$

To complete the converse: Take any positive and vanishing sequence $\{\epsilon\} \rightarrow 0$. Consider the corresponding sequence of list codes (with increasing blocklengths $n_{\mathrm{s}}$ and $n_{\mathrm{c}}$ ) and time-shared pmfs $\left\{P_{\tilde{W}}\right\}$. Since $\mathcal{W}$ is a finite alphabet, by the Bolzano-Weierstrass theorem, $\left\{P_{\tilde{W}}\right\}$ will contain a convergent subsequence with respect to the variational distance. Let $P_{\tilde{W}}^{*}$ denote the limit of the convergent subsequence and $\tilde{W}^{*} \sim P_{\tilde{W}}^{*}$. We then have $\kappa I\left(\tilde{W}^{*} ; U_{k}\right) \geq H\left(X \mid Y_{k}\right)-D_{k}$ by the continuity of mutual information [32, Sec. 2.3]. 


\section{Proof of Lemma 3 - Achievability}

We restrict attention to the bandwidth matched case $(\kappa=1$ and $n_{\mathrm{s}}=n_{\mathrm{c}}=n$ ), to help simplify notation and elucidate the main ideas of the achievability proof. Extending this proof to the bandwidth mismatched case is relatively easy, because we will use separate source and channel codebooks and the error probability bounds depend only on the marginal source and channel distributions.

\section{A. Notation and Letter-Typical Sets}

For any given random variable $\omega$ and set $\Omega$, let us denote the indicator function for the event that $\omega$ falls in $\Omega$ by

$$
\mathbb{1}\{\omega \in \Omega\}:= \begin{cases}1 & \text { if } \omega \in \Omega \\ 0 & \text { otherwise. }\end{cases}
$$

The proof will use letter typical sets [33]. Consider a pair of random variables $(A, B) \sim P_{A, B}$ on $\mathcal{A} \times \mathcal{B}$, where $\mathcal{A}$ and $\mathcal{B}$ are finite alphabets. Let $P_{A}$ denote the marginal pmf of $A$. For $\epsilon>0$ and a positive integer $n$, the typical set of $P_{A}$ is

$$
\begin{aligned}
\mathcal{T}_{\epsilon}{ }^{n}\left(P_{A}\right): & =\left\{\mathbb{a} \in \mathcal{A}^{n}:\right. \\
& \left.\left|\frac{1}{n} N\left(a^{\prime} \mid \mathbb{a}\right)-P_{A}\left(a^{\prime}\right)\right| \leq \epsilon P_{A}\left(a^{\prime}\right), \quad \forall a^{\prime} \in \mathcal{A}\right\},
\end{aligned}
$$

where $N\left(a^{\prime} \mid \mathbb{a}\right)$ represents the number of occurrences of $a^{\prime}$ in the sequence $\mathbb{a}$. The jointly typical set of $P_{A, B}$ is

$$
\begin{aligned}
\mathcal{T}_{\epsilon}^{n}\left(P_{A, B}\right):= & \left\{(\mathfrak{a}, \mathfrak{b}) \in \mathcal{A}^{n} \times \mathcal{B}^{n}: \mid \frac{1}{n} N\left(a^{\prime}, b^{\prime} \mid \mathfrak{a}, \mathfrak{b}\right)\right. \\
& \left.-P_{A, B}\left(a^{\prime}, b^{\prime}\right) \mid \leq \epsilon P_{A, B}\left(a^{\prime}, b^{\prime}\right), \quad \forall\left(a^{\prime}, b^{\prime}\right)\right\} .
\end{aligned}
$$

The conditionally typical set of $P_{A, B}$ given $\mathbb{b} \in \mathcal{B}^{n}$ is

$$
\mathcal{T}_{\epsilon}^{n}\left(P_{A, B} \mid \mathbb{b}\right):=\left\{\mathbb{a} \in \mathcal{A}^{n}:(\mathbb{a}, \mathfrak{b}) \in \mathcal{T}_{\epsilon}^{n}\left(P_{A, B}\right)\right\} .
$$

The proof will frequently use the property that joint typicality implies marginal typicality,

$$
(\mathbb{a}, \mathfrak{b}) \in \mathcal{T}_{\epsilon}^{n}\left(P_{A, B}\right) \Rightarrow \mathbb{a} \in \mathcal{T}_{\epsilon}^{n}\left(P_{A}\right) \text { and } \mathfrak{b} \in \mathcal{T}_{\epsilon}^{n}\left(P_{B}\right),
$$

and the following lemmas. Let

$$
\mu_{A}:=\min _{a \in \operatorname{supp}\left(P_{A}\right)} P_{A}(a),
$$

and

$$
\mu_{A, B}:=\min _{(a, b) \in \operatorname{supp}\left(P_{A, B}\right)} P_{A, B}(a, b),
$$

where $\operatorname{supp}(\cdot)$ denotes the support set of the indicated distribution.

Lemma 4: If $\mathbb{A}:=\left(A_{1}, A_{2}, \ldots, A_{n}\right)$ is generated iid with $P_{A}, 0<\epsilon \leq \mu_{A}$ and $₫ \in \mathcal{T}_{\epsilon}^{n}\left(P_{A}\right)$, then [33. Thm. 1.1]

$$
2^{-n H(A)(1+\epsilon)} \leq \mathbb{P}[\mathbb{A}=\mathbb{a}] \leq 2^{-n H(A)(1-\epsilon)}
$$

and

$$
1-2|\mathcal{A}| \exp \left(-n \epsilon^{2} \mu_{A}\right) \leq \mathbb{P}\left[\mathbb{A} \in \mathcal{T}_{\epsilon}^{n}\left(P_{A}\right)\right] \leq 1 .
$$

Lemma 5: If $\mathbb{A}:=\left(A_{1}, A_{2}, \ldots, A_{n}\right)$ is generated iid with $P_{A}, 0<\epsilon_{1}<\epsilon \leq \mu_{A B}$ and $\mathfrak{b} \in \mathcal{T}_{\epsilon_{1}}^{n}\left(P_{B}\right)$, then [33, Thm. 1.3]

$$
\mathbb{P}\left[\mathbb{A} \in \mathcal{T}_{\epsilon}^{n}\left(P_{A, B} \mid \mathbb{b}\right)\right] \leq 2^{-n(I(A ; B)-2 \epsilon H(A))}
$$

and

$$
\mathbb{P}\left[\mathbb{A} \in \mathcal{T}_{\epsilon}^{n}\left(P_{A, B} \mid \mathbb{b}\right)\right] \geq\left(1-\zeta_{n}\right) 2^{-n(I(A ; B)+2 \epsilon H(A))},
$$

where 9

$$
\zeta_{n}:=2|\mathcal{A}||\mathcal{B}| \exp \left(-2 n\left(1-\epsilon_{1}\right) \frac{\left(\epsilon-\epsilon_{1}\right)^{2}}{1+\epsilon_{1}} \mu_{A B}^{2}\right) .
$$

\section{B. Distributions and Typicality Constants}

Pick any pmf $P_{W}$ on $\mathcal{W}$. Let

$$
X \sim P_{X}, \quad\left(X, Y_{k}\right) \sim P_{X, Y_{k}} \quad \text { and } \quad\left(W, U_{k}\right) \sim P_{W, U_{k}}
$$

denote the pmfs of the indicated variables. Fix any arbitrarily small constants $\epsilon, \epsilon_{1}, \delta$ and $\delta_{1}$ satisfying

$$
\begin{array}{r}
0<\delta_{1}<\delta<\min _{k} \mu_{W, U_{k}} \\
\text { and } 0<\epsilon_{1}<\epsilon<\min _{k} \mu_{X, Y_{k}},
\end{array}
$$

with

$$
\epsilon<\frac{\min _{k} \mu_{W, U_{k}}}{2 H(X) \ln 2} \delta^{2}
$$

\section{Code Construction and Encoding}

The encoder mirrors that of [8, Thm. 6]. Randomly generate a source codebook $\mathcal{C}_{X}$ with

$$
M=\left\lfloor 2^{n H(X)(1+\epsilon)}\right\rfloor
$$

codewords, each of length $n$, by selecting symbols from $\mathcal{X}$ in an iid fashion using $P_{X}$ :

$$
\mathcal{C}_{X}:=\left\{\mathbb{X}(m)=\left(X_{1}(m), X_{2}(m), \ldots, X_{n}(m)\right)\right\}_{m=1}^{M} .
$$

In the same way, generate a channel codebook $\mathcal{C}_{W}$ with $M$ codewords of length $n$ using $P_{W}$ :

$$
\mathcal{C}_{W}:=\left\{\mathbb{W}(m)=\left(W_{1}(m), W_{2}(m), \ldots, W_{n}(m)\right)\right\}_{m=1}^{M} .
$$

Upon observing the source $\mathbb{X}$, the transmitter searches through the source codebook $\mathcal{C}_{X}$ for the smallest index $m$ such that $\mathbb{X}=\mathbb{X}(m)$. If successful, the transmitter sends the corresponding channel codeword $\mathbb{W}(m)$; and, if unsuccessful, it sends $\mathbb{W}$ generated iid $\sim P_{W}$.

\section{List Decoding at Receiver $k$}

The decoder (and error analysis) differ from [8, Thm. 6]. Upon observing the channel output $\mathbb{U}_{k}$ and side information $\mho_{k}$, receiver $k$ outputs the list

$$
\begin{aligned}
\mathcal{L}_{k}:=\left\{\mathbb{X}(m) \in \mathcal{C}_{X}:\left(\mathbb{X}(m), \mathbb{Y}_{k}\right) \in \mathcal{T}_{\epsilon}^{n}\left(P_{X, Y_{k}}\right)\right. \\
\left.\quad \text { and }\left(\mathbb{W}(m), \mathbb{U}_{k}\right) \in \mathcal{T}_{\delta}^{n}\left(P_{W, U_{k}}\right)\right\} .
\end{aligned}
$$

An error is declared at receiver $k$ if the source is not in the list $\mathcal{X} \notin \mathcal{L}_{k}$ or the list is too large

$$
\left|\mathcal{L}_{k}\right|>2^{n D_{k}} .
$$

\footnotetext{
${ }^{9}$ Here we use I. Sason's correction to 33. Thm. 1.3], see 34 pp. 140-154].
} 


\section{E. Error Analysis: Decoding error event $\mathcal{E}$}

Denote the event of an error at any receiver by

$$
\mathcal{E}:=\bigcup_{k=1}^{K}\left(\left\{\mathbb{X} \notin \mathcal{L}_{k}\right\} \cup\left\{\left|\mathcal{L}_{k}\right|>2^{n D_{k}}\right\}\right) .
$$

By the union bound,

$$
\mathbb{P}[\mathcal{E}] \leq \sum_{k=1}^{K}\left(\mathbb{P}\left[\mathbb{X} \notin \mathcal{L}_{k}\right]+\mathbb{P}\left[\left|\mathcal{L}_{k}\right|>2^{n D_{k}}\right]\right) .
$$

In the following subsections, we show that the average error probability $\mathbb{P}[\mathcal{E}]$ satisfies

$$
\mathbb{P}[\mathcal{E}] \leq b 2^{-a n}
$$

for some finite $a, b>0$, whenever $\epsilon$ and $\delta$ satisfy (18) and

$$
D_{k}>\max \left\{H\left(X \mid Y_{k}\right)-I\left(W ; U_{k}\right), 0\right\}, \quad \forall k .
$$

Therefore, for any $\epsilon^{*}>0$ there exists an $\left(n, L_{1}, L_{2}, \ldots, L_{K}\right)$ list code such that $\mathbb{P}\left[\mathbb{X} \notin \mathcal{L}_{k}\right] \leq \epsilon^{*}$ and $\left|\mathcal{L}_{k}\right| \leq 2^{n D_{k}}$ for all $k$.

The remainder of this section is devoted to proving 23 . The derivation of the bound is a little tedious and the reader needs only (23) to proceed to the achievability proofs of Theorems 1 and 2 in Sections $\mathrm{VIII}$ and $\mathrm{X}$ respectively.

F. Error Analysis: Probability $\backslash$ is not in the source codebook

The probability that the source is not in the source codebook $\mathbb{P}\left[\mathbb{X} \notin \mathcal{C}_{X}\right]$ is bounded by

$$
\mathbb{P}\left[\mathbb{X} \notin \mathcal{C}_{X}\right] \leq b_{1} 2^{-a_{1} n},
$$

where

$$
a_{1}:=\min \left\{\epsilon_{1}^{2} \mu_{X},\left(H(X) \cdot\left(\epsilon-\epsilon_{1}\right)\right)\right\} / \ln 2
$$

and $b_{1}:=2|\mathcal{X}|+1$ are both positive by (18).

The steps leading to 24 are

$$
\begin{aligned}
& \mathbb{P}\left[\mathbb{X} \notin \mathcal{C}_{X}\right] \\
& \leq \mathbb{P}\left[\mathbb{X} \notin \mathcal{T}_{\epsilon_{1}}^{n}\left(P_{X}\right)\right]+\mathbb{P}\left[\mathbb{X} \notin \mathcal{C}_{X} \mid \mathbb{X} \in \mathcal{T}_{\epsilon_{1}}^{n}\left(P_{X}\right)\right] \\
& \stackrel{\mathrm{a}}{\leq} 2|\mathcal{X}| e^{-n \epsilon_{1}^{2} \mu_{X}}+\mathbb{P}\left[\bigcap_{m=1}^{M}\{\mathbb{X}(m) \neq \mathbb{X}\} \mid \mathbb{X} \in \mathcal{T}_{\epsilon_{1}}^{n}\left(P_{X}\right)\right] \\
& \stackrel{\mathrm{b}}{=} 2|\mathcal{X}| e^{-n \epsilon_{1}^{2} \mu_{X}}+\prod_{m=1}^{M}\left(1-\mathbb{P}\left[\mathfrak{X}(m)=\mathbb{X} \mid \mathbb{X} \in \mathcal{T}_{\epsilon_{1}}^{n}\left(P_{X}\right)\right]\right) \\
& \stackrel{\mathrm{c}}{\leq} 2|\mathcal{X}| e^{-n \epsilon_{1}^{2} \mu_{X}}+\left(1-2^{-n H(X)\left(1+\epsilon_{1}\right)}\right) \\
& \stackrel{\mathrm{d}}{\leq} 2|\mathcal{X}| e^{-n \epsilon_{1}^{2} \mu_{X}}+\exp \left(-M 2^{-n H(X)\left(1+\epsilon_{1}\right)}\right) \\
& \mathrm{e} 2|\mathcal{X}| e^{-n \epsilon_{1}^{2} \mu_{X}}+\exp \left(-2^{n H(X)\left(\epsilon-\epsilon_{1}\right)}\right) .
\end{aligned}
$$

Notes:
a. apply Lemma 4 ,
b. the codewords in $\mathcal{C}_{X}$ are independent;
c. use Lemma 4 with $\mathbb{X}(m)$ iid $\sim P_{X}$;
d. use the inequality

$$
(1-c)^{M} \leq e^{-c M}, \quad \forall M \geq 1, c \in[0,1] ; \text { and }
$$

e. bound $M$ via (19).

The bound in (24) follows since $H(X)\left(\epsilon-\epsilon_{1}\right)>0$ from (18).

G. Error Analysis: Probability $\backslash$ is not in receiver $k$ 's list $\mathcal{L}_{k}$

Consider the probability that the source $\mathcal{X}$ is not in receiver $k$ 's list $\mathcal{L}_{k}$. We have

$$
\begin{aligned}
\mathbb{P}\left[\mathfrak{X} \notin \mathcal{L}_{k}\right] \leq \mathbb{P}\left[\mathbb{X} \notin \mathcal{C}_{X}\right] \\
+\mathbb{P}\left[\left(\mathbb{K}, \mathbb{Y}_{k}\right) \notin \mathcal{T}_{\epsilon_{1}}\left(P_{X, Y_{k}}\right)\right]+\mathbb{P}\left[\mathcal{S}_{1}\right]
\end{aligned}
$$

$$
\begin{aligned}
& \mathbb{P}\left[\mathcal{S}_{1}\right] \stackrel{\mathrm{a}}{=} \mathbb{P}\left[\left\{\mathbb{X} \notin \mathcal{L}_{k}\right\} \cap\left\{\left(\mathbb{X}, Y_{k}\right) \in \mathcal{T}_{\epsilon_{1}}^{n}\left(P_{X Y_{k}}\right)\right\} \cap\left(\bigcup_{m=1}^{M}\left\{\mathbb{X} \neq \mathbb{X}\left(m^{\prime}\right), \forall m^{\prime}<m\right\} \cap\{\mathbb{X}=\mathbb{X}(m)\}\right)\right] \\
& \stackrel{\mathrm{b}}{\leq} \sum_{m=1}^{M} \mathbb{P}\left[\left\{\mathbb{X} \notin \mathcal{L}_{k}\right\} \cap\left\{\left(\mathbb{X}, \mathbb{Y}_{k}\right) \in \mathcal{T}_{\epsilon_{1}}^{n}\left(P_{X Y_{k}}\right)\right\} \cap\left\{\mathbb{X} \neq \mathbb{X}\left(m^{\prime}\right), \forall m^{\prime}<m\right\} \cap\{\mathbb{X}=\mathbb{X}(m)\}\right] \\
& \stackrel{\mathrm{c}}{\leq} \sum_{m=1}^{M} \mathbb{P}\left[\left\{\mathbb{X} \neq \mathbb{X}\left(m^{\prime}\right), \forall m^{\prime}<m\right\} \cap\{\mathbb{X}=\mathbb{X}(m)\} \mid\left(\mathbb{X}, \mathbb{Y}_{k}\right) \in \mathcal{T}_{\epsilon_{1}}^{n}\left(P_{X, Y_{k}}\right)\right] \\
& \mathbb{P}\left[\mathbb{X} \notin \mathcal{L}_{k} \mid\left\{\left(\mathbb{X}, \mathbb{Y}_{k}\right) \in \mathcal{T}_{\epsilon_{1}}^{n}\left(P_{X Y_{k}}\right)\right\} \cap\left\{\mathbb{X} \neq \mathbb{X}\left(m^{\prime}\right), \forall m^{\prime}<m\right\} \cap\{\mathbb{X}=\mathbb{X}(m)\}\right] \\
& \stackrel{\mathrm{d}}{\leq} \sum_{m=1}^{M} \mathbb{P}\left[\mathbb{X}=\mathbb{X}(m) \mid \mathfrak{X} \in \mathcal{T}_{\epsilon_{1}}^{n}\left(P_{X}\right)\right] \mathbb{P}\left[\left(\mathbb{W}, \mathbb{U}_{k}\right) \notin \mathcal{T}_{\delta}^{n}\left(P_{W}, U_{k}\right) \mid\left\{\left(\mathbb{X}, \mathbb{Y}_{k}\right) \in \mathcal{T}_{\epsilon_{1}}^{n}\left(P_{X Y_{k}}\right)\right\}\right. \\
& \left.\cap\left\{\mathbb{X} \neq \mathbb{X}\left(m^{\prime}\right), \forall m^{\prime}<m\right\} \cap\{\mathbb{X}=\mathbb{X}(m)\} \cap\{\mathbb{W}=\mathbb{W}(m)\}\right] \\
& \stackrel{\mathrm{e}}{=} \sum_{m=1}^{M} \mathbb{P}\left[\mathbb{X}=\mathbb{X}(m) \mid \mathbb{X} \in \mathcal{T}_{\epsilon_{1}}^{n}\left(P_{X}\right)\right] \mathbb{P}\left[\left(\mathbb{W}, \mathbb{U}_{k}\right) \notin \mathcal{T}_{\delta}^{n}\left(P_{W, U_{k}}\right)\right] \\
& \stackrel{\mathrm{f}}{\leq} M 2|\mathcal{W}|\left|\mathcal{U}_{k}\right| 2^{-n H(X)\left(1-\epsilon_{1}\right)} 2^{-n \frac{\delta^{2} \mu_{W, U_{k}}}{\ln 2}} \\
& \stackrel{\mathrm{g}}{\leq} 2|\mathcal{W}|\left|\mathcal{U}_{k}\right| 2^{+2 \epsilon n H(X)} 2^{-n \frac{\delta^{2} \mu_{W, U_{k}}}{\ln 2}} \text {. }
\end{aligned}
$$


where

$\mathcal{S}_{1}:=\left\{\mathbb{X} \notin \mathcal{L}_{k}\right\} \cap\left\{\left(\mathcal{X}, \mathbb{Y}_{k}\right) \in \mathcal{T}_{\epsilon_{1}}^{n}\left(P_{X Y_{k}}\right)\right\} \cap\left\{\mathbb{X} \in \mathcal{C}_{X}\right\}$.

The probability $\mathbb{P}\left[\mathcal{S}_{1}\right]$ is bounded from above by

$$
\mathbb{P}\left[\mathcal{S}_{1}\right] \leq 2|\mathcal{W}|\left|\mathcal{U}_{k}\right| 2^{-a_{2} n}
$$

where

$$
a_{2}:=\delta^{2} \mu_{W, U_{k}} / \ln 2-2 \epsilon_{1} H(X)
$$

is positive by (18). The steps leading to (27) are described above in (28). Notes for 28):

a. expand the event that the source $\mathbb{X}$ appears in $\mathcal{C}_{X}$;

b. union bound;

c. Bayes' law and $\mathbb{P}\left[\left(\mathbb{X}, \mathbb{Y}_{k}\right) \in \mathcal{T}_{\epsilon}^{n}\left(P_{X Y_{k}}\right)\right] \leq 1$;

d. conditioned on $\left(\mathcal{X}, \mathbb{Y}_{k}\right)$ typical, $\mathbb{X}=\mathbb{X}(m)$ and $\mathbb{W}=$ $\mathbb{W}(m)$, the error $\mathbb{X} \notin \mathcal{L}_{k}$ occurs if and only if $\left(\mathbb{W}, \mathbb{U}_{k}\right)$ are not jointly typical;

e. the source and channel codebooks are independent, and all channel codewords are constructed in the same way;

f. Lemma 4, and

g. bound the codebook cardinality $M$ as in (19).

Combining (26) and 27) with 24) and Lemma 4, we have

$$
\mathbb{P}\left[\mathbb{X} \notin \mathcal{L}_{k}\right] \leq b_{3} 2^{-a_{3} n},
$$

for some finite $a_{3}, b_{3}>0$.

\section{H. Error Analysis: Probability receiver $k$ 's list $\mathcal{L}_{k}$ is too large}

Now consider the probability that the size of list $\mathcal{L}_{k}$ is too large. We start with

$$
\begin{aligned}
\mathbb{P}\left[\left|\mathcal{L}_{k}\right|>2^{n D_{k}}\right] \leq \mathbb{P}\left[\mathbb{X} \notin \mathcal{C}_{X}\right]+\mathbb{P}\left[\left(\mathcal{X}, \mathbb{Y}_{k}\right) \notin \mathcal{T}_{\epsilon_{1}}^{n}\right] \\
+\mathbb{P}\left[\left(\mathbb{W}, \mathbb{U}_{k}\right) \notin \mathcal{T}_{\delta_{1}}^{n}\right] \\
+\mathbb{P}\left[\left|\mathcal{L}_{k}\right|>2^{n D_{k}} \mid \mathcal{S}_{2}\right]
\end{aligned}
$$

where

$$
\mathcal{S}_{2}:=\left\{\mathbb{X} \in \mathcal{C}_{X}\right\} \cap\left\{\left(\mathbb{X}, \mathbb{V}_{k}\right) \in \mathcal{T}_{\epsilon_{1}}^{n}\right\} \cap\left\{\left(\mathbb{W}, \mathbb{U}_{k}\right) \in \mathcal{T}_{\delta_{1}}^{n}\right\},
$$

and $\mathcal{T}_{\epsilon_{1}}^{n}\left(P_{X, Y_{k}}\right)$ and $\mathcal{T}_{\delta_{1}}^{n}\left(P_{W, U_{k}}\right)$ have been abbreviated by $\mathcal{T}_{\epsilon_{1}}^{n}$ and $\mathcal{T}_{\delta_{1}}^{n}$ respectively. Apply Markov's inequality to the rightmost probability in 30 to get

$$
\mathbb{P}\left[\left|\mathcal{L}_{k}\right|>2^{n D_{k}} \mid \mathcal{S}_{2}\right] \leq 2^{-n D_{k}} \mathbb{E}\left[\left|\mathcal{L}_{k}\right| \mid \mathcal{S}_{2}\right],
$$

where the expectation is understood to be

$$
\mathbb{E}\left[\left|\mathcal{L}_{k}\right| \mid \mathcal{S}_{2}\right]:=\sum_{l} l \cdot \mathbb{P}\left[\left|\mathcal{L}_{k}\right|=l \mid \mathcal{S}_{2}\right] .
$$

We now expand the above expectation over $\mathbb{X} \in \mathcal{C}_{X}$ (the $M$ possible encodings of $\mathbb{X}$ ) to get

$$
\begin{aligned}
& \mathbb{E}\left[\left|\mathcal{L}_{k}\right| \mid \mathcal{S}_{2}\right] \\
& =\sum_{m=1}^{M} \mathbb{E}\left[\left|\mathcal{L}_{k}\right| \mid \mathcal{S}_{2} \cap\left\{\mathbb{X} \neq \mathbb{X}\left(m^{\prime}\right), \forall m^{\prime}<m\right\} \cap\{\mathbb{X}=\mathbb{X}(m)\}\right] \\
& \quad \cdot \mathbb{P}\left[\left\{\mathbb{X} \neq \mathbb{X}\left(m^{\prime}\right), \forall m^{\prime}<m\right\} \cap\{\mathbb{X}=\mathbb{X}(m)\} \mid \mathcal{S}_{2}\right] . \text { (32) }
\end{aligned}
$$

Consider the expectation on the right hand side of (32). Let

$$
\mathcal{S}_{2, m}:=\mathcal{S}_{2} \cap\left\{\mathbb{X} \neq \mathbb{X}\left(m^{\prime}\right), \forall m^{\prime}<m\right\} \cap\{\mathbb{X}=\mathbb{X}(m)\} .
$$

We have

$$
\begin{aligned}
\mathbb{E} & {\left[\left|\mathcal{L}_{k}\right| \mid \mathcal{S}_{2, m}\right] } \\
= & \mathbb{E}\left[\sum_{\tilde{m}=1}^{M} \mathbb{1}\left\{\left(\mathbb{X}(\tilde{m}), \mathbb{\mho}_{k}\right) \in \mathcal{T}_{\epsilon}^{n}\right\} \mathbb{1}\left\{\left(\mathbb{W}(\tilde{m}), \mathbb{U}_{k}\right) \in \mathcal{T}_{\delta}^{n}\right\} \mid \mathcal{S}_{2, m}\right] \\
= & \sum_{\tilde{m}=1}^{M} \mathbb{P}\left[\left\{\left(\mathbb{X}(\tilde{m}), \mathbb{Y}_{k}\right) \in \mathcal{T}_{\epsilon}^{n}\right\} \cap\left\{\left(\mathbb{W}(\tilde{m}), \mathbb{U}_{k}\right) \in \mathcal{T}_{\delta}^{n}\right\} \mid \mathcal{S}_{2, m}\right] \\
= & \sum_{\tilde{m}=1}^{M} \mathbb{P}\left[\left(\mathbb{X}(\tilde{m}), \mathbb{Y}_{k}\right) \in \mathcal{T}_{\epsilon}^{n} \mid \mathcal{S}_{2, m}\right] \\
& \cdot \mathbb{P}\left[\left(\mathbb{W}(\tilde{m}), \mathbb{U}_{k}\right) \in \mathcal{T}_{\delta}^{n} \mid \mathcal{S}_{2, m} \cap\left\{\left(\mathbb{X}(\tilde{m}), \mathbb{Y}_{k}\right) \in \mathcal{T}_{\epsilon}^{n}\right\}\right],
\end{aligned}
$$

where $\mathcal{T}_{\epsilon}^{n}\left(P_{X, Y_{k}}\right)$ and $\mathcal{T}_{\delta}^{n}\left(P_{W, U_{k}}\right)$ have been abbreviated by $\mathcal{T}_{\epsilon}^{n}$ and $\mathcal{T}_{\delta}^{n}$ respectively.

The event $\mathcal{S}_{2, m}$ implies that the source $\mathbb{X}$ is equal to the $m$-th source codeword $\mathbb{X}(m)$ and $\mathbb{W}=\mathbb{W}(m)$ is sent over the channel. We now bound the two probabilities on the right hand side of (33) separately for each of the three cases $1 \leq \tilde{m}<m$, $\tilde{m}=m$ and $m<\tilde{m} \leq M$.

Case $1(1 \leq \tilde{m}<m)$ : The first probability in (33) is bounded by

$$
\begin{aligned}
& \mathbb{P}\left[\left(\mathbb{X}(\tilde{m}), \mathbb{Y}_{k}\right) \in \mathcal{T}_{\epsilon}^{n} \mid \mathcal{S}_{2, m}\right] \\
& \quad \stackrel{\mathrm{a}}{=} \mathbb{P}\left[\left(\mathbb{X}(\tilde{m}), \mathbb{Y}_{k}\right) \in \mathcal{T}_{\epsilon}^{n} \mid\left\{\left(\mathbb{X}, \mathbb{Y}_{k}\right) \in \mathcal{T}_{\epsilon_{1}}^{n}\right\} \cap\{\mathbb{X} \neq \mathbb{X}(\tilde{m})\}\right] \\
& \quad \stackrel{\mathrm{b}}{\leq} \frac{\mathbb{P}\left[\left(\mathbb{X}(\tilde{m}), \mathbb{Y}_{k}\right) \in \mathcal{T}_{\epsilon}^{n}\left(P_{X, Y_{k}}\right) \mid \mathbb{Y}_{k} \in \mathcal{T}_{\epsilon_{1}}^{n}\left(P_{Y_{k}}\right)\right]}{\mathbb{P}\left[\mathbb{X} \neq \mathbb{X}(\tilde{m}) \mid \mathbb{X} \in \mathcal{T}_{\epsilon_{1}}^{n}\left(P_{X}\right)\right]} \\
& \quad \stackrel{\mathrm{c}}{\leq} \alpha_{n} 2^{-n\left(I\left(X ; Y_{k}\right)-2 \epsilon H(X)\right)} .
\end{aligned}
$$

Notes:

a. codewords and codebook are generated independently;

b. Bayes' law and the trivial bound $\mathbb{P}[\mathbb{X} \neq \mathbb{X}(\tilde{m}) \mid\{(\mathbb{X}(\tilde{m})$, $\left.\left.\left.\mathbb{Y}_{k}\right) \in \mathcal{T}_{\epsilon}^{n}\left(P_{X, Y_{k}}\right)\right\} \cap\left\{\left(\mathbb{X}, \mathbb{Y}_{k}\right) \in \mathcal{T}_{\epsilon_{1}}^{n}\right\}\right] \leq 1$; and

c. apply Lemmas 4 and 5 respectively to the denominator and numerator in step (b) and set

$$
\alpha_{n}:=\frac{\exp \left(2^{n H(X)\left(1-\epsilon_{1}\right)}\right)}{\exp \left(2^{n H(X)\left(1-\epsilon_{1}\right)}\right)-1} .
$$

Similarly, by Lemma 5 the rightmost probability in (33) is bounded by

$$
\begin{aligned}
\mathbb{P}\left[\left(\mathbb{W}(\tilde{m}), \mathbb{U}_{k}\right) \in \mathcal{T}_{\delta}^{n} \mid \mathcal{S}_{2, m}\right. & \left.\cap\left\{\left(\mathbb{X}(\tilde{m}), \mathbb{Y}_{k}\right) \in \mathcal{T}_{\epsilon}^{n}\right\}\right] \\
\leq & 2^{-n\left(I\left(W ; U_{k}\right)-2 \delta H(W)\right)} .
\end{aligned}
$$

Case $2(\tilde{m}=m)$ : Bound both probabilities in 33 by one.

Case $3(m<\tilde{m} \leq M)$ : Conditioned on $\mathcal{S}_{2, m}$, the encoder has only considered the codewords $\mathbb{X}(1), \ldots, \mathbb{X}(m)$. Thus, even conditional on $\mathcal{S}_{2, m}$, the codewords thereafter $\mathbb{X}(m+1), \ldots, \mathbb{X}(M)$ are independent iid $\sim P_{X}$ sequences. From Lemma 5 ,

$$
\mathbb{P}\left[\left(\mathcal{X}(\tilde{m}), \mathbb{Y}_{k}\right) \in \mathcal{T}_{\epsilon}^{n} \mid \mathcal{S}_{2, m}\right] \leq 2^{-n\left(I\left(X ; Y_{k}\right)-2 \epsilon H(X)\right)},
$$

Similarly,

$$
\begin{aligned}
\mathbb{P}\left[\left(\mathbb{W}(\tilde{m}), \mathbb{U}_{k}\right) \in \mathcal{T}_{\delta}^{n} \mid \mathcal{S}_{2, m}\right. & \left.\cap\left\{\left(\mathbb{X}(\tilde{m}), \mathbb{Y}_{k}\right) \in \mathcal{T}_{\epsilon}^{n}\right\}\right] \\
& \leq 2^{-n\left(I\left(W ; U_{k}\right)-2 \delta H(W)\right)} .
\end{aligned}
$$


Collectively, (19) and 33) to 38) imply

$$
\begin{aligned}
& \mathbb{E}\left[\left|\mathcal{L}_{k}\right| \mid \mathcal{S}_{2, m}\right] \\
& \leq 1+\alpha_{n} 2^{n\left(H\left(X \mid Y_{k}\right)-I\left(W ; U_{k}\right)\right)} 2^{n(3 \epsilon H(X)+2 \delta H(W)) .}
\end{aligned}
$$

Combine (30), 31] and 39) to get

$$
\begin{aligned}
& \mathbb{P}\left[\left|\mathcal{L}_{k}\right|>2^{n D_{k}}\right] \\
& \leq \mathbb{P}\left[\mathbb{X} \notin \mathcal{C}_{X}\right]+\mathbb{P}\left[\left(\mathcal{X}, \mho_{k}\right) \notin \mathcal{T}_{\epsilon}^{n}\right]+\mathbb{P}\left[\left(\mathbb{W}, \mathbb{U}_{k}\right) \notin \mathcal{T}_{\delta}^{n}\right] \\
& \quad+\alpha_{n} 2^{-n\left(D_{k}-H\left(X \mid Y_{k}\right)+I\left(W ; U_{k}\right)\right)} 2^{n(3 \epsilon H(X)+2 \delta H(W))} \\
& \quad+2^{-n D_{k}} .
\end{aligned}
$$

Lemma 4 and 24) imply

$$
\mathbb{P}\left[\left|\mathcal{L}_{k}\right|>2^{n D_{k}}\right] \leq b_{4} 2^{-a_{4} n},
$$

for some finite $a_{4}, b_{4}>0$ whenever

$D_{k}>\max \left\{H\left(X \mid Y_{k}\right)-I\left(W ; U_{k}\right), 0\right\}+3 \epsilon H(X)+2 \delta H(W)$

and $\epsilon, \epsilon_{1}, \delta$ and $\delta_{1}$ satisfy 18 . The result follows because $\epsilon$ and $\delta$ can be chosen arbitrarily small and $H(X)$ and $H(W)$ are finite.

\section{Proof of Theorem 1 - Converse}

Fix $\epsilon>0$. Consider any $\left(n_{\mathrm{s}}, n_{\mathrm{c}}, R_{1}, R_{2}, \ldots, R_{K}\right)$-code with $\mathbb{P}\left[\hat{\mathcal{K}}_{k} \neq \mathbb{X}\right] \leq \epsilon$ for all $k$. Recall the timeshared pmf $P_{\tilde{W}}$ on $\mathcal{W}$, defined in (15). Let

$$
\left(\tilde{W}, \tilde{U}_{1}, \tilde{U}_{2}, \ldots, \tilde{U}_{K}\right) \sim P_{\tilde{W}}(\cdot) T(\cdot \cdot \cdot)
$$

and $\tilde{V}_{k}=\phi_{k}(\tilde{W})$. Mirroring the steps of Section V

$$
\begin{aligned}
n_{\mathrm{c}} I\left(\tilde{W} ; \tilde{U}_{k}, \tilde{V}_{k}\right) & \geq I\left(\mathbb{W} ; \mathbb{U}_{k}, \mathbb{V}_{k}\right) \stackrel{\mathrm{a}}{\geq} I\left(\mathbb{W} ; \mathbb{U}_{k}, M_{k}\right) \\
& \geq I\left(\mathbb{X} ; \mathbb{U}_{k}, M_{k} \mid \mathbb{Y}_{k}\right) \\
& =n_{\mathrm{s}} H\left(X \mid Y_{k}\right)-H\left(\mathbb{X} \mid \mathbb{Y}_{k}, \mathbb{U}_{k}, M_{k}\right),
\end{aligned}
$$

where (a) and (b) use $M_{k} \leftrightarrow\left(\mathbb{U}_{k}, \mathbb{V}_{k}\right) \leftrightarrow \mathbb{W}$ and $\left(\mathbb{X}, \mathbb{V}_{k}\right) \leftrightarrow$ $\mathbb{W} \leftrightarrow\left(\mathbb{U}_{k}, M_{k}\right)$. Similarly,

$$
\begin{aligned}
n_{\mathrm{c}} I\left(\tilde{W} ; \tilde{U}_{k}\right) & +n_{\mathrm{s}} R_{k} \\
& \geq I\left(\mathbb{W} ; \mathbb{U}_{k}\right)+H\left(M_{k} \mid \mathbb{U}_{k}\right) \\
& \geq I\left(\mathbb{W} ; \mathbb{U}_{k}, M_{k}\right) \\
& \geq n_{\mathrm{s}} H\left(X \mid Y_{k}\right)-H\left(\mathbb{X} \mid \mathbb{U}_{k}, \mathbb{Y}_{k}, M_{k}\right) .
\end{aligned}
$$

After applying Fano's inequality [23, Thm. 2.10.1] to $H\left(\mathbb{X} \mid \cup_{k}, Y_{k}, M_{k}\right)$ in (42) and (43), the converse follows in the same way as the closing of Section $\mathrm{V}$.

\section{Proof of Theorem 1- Achievability}

We now present an achievability proof for the bandwidth matched case, where $\kappa=1$ and $n_{\mathrm{s}}=n_{\mathrm{c}}=n$. The mismatched bandwidth case follows by similar arguments. Our approach to the proof combines the list decoder of Section VI with hash-and-forward coding at the helpers.

\section{A. Code Construction}

Fix a pmf $P_{W}$ on $\mathcal{W}$ and let us assume that for all $k$

$$
H\left(X \mid Y_{k}\right)<I\left(W ; U_{k}\right)+\min \left\{R_{k}, I\left(W ; V_{k} \mid U_{k}\right)\right\}
$$

and

$$
H\left(X \mid Y_{k}\right) \geq I\left(W ; U_{k}\right) .
$$

The assumption above (44) matches that in Theorem 1 and (45) ensures that every receiver requires a positive helper rate to reliably decode the source $\backslash$. At the end of the proof, we will relax (45) to include situations where some receivers don't require a positive helper rate, i.e., $H\left(X \mid Y_{k}\right)<I\left(W ; U_{k}\right)$ for some $k$.

Generate a random list code, as described in Section VI with the parameters described above, and let $\mathcal{C}_{X}$ and $\mathcal{C}_{W}$ denote the source and channel codebooks respectively. Fix $\epsilon, \epsilon_{1}, \delta$ and $\delta_{1}$ arbitrarily small, but always satisfying (18). For each receiver $k$, choose any list exponent $D_{k}$ satisfying

$$
\begin{aligned}
H\left(X \mid Y_{k}\right)-I\left(W ; U_{k}\right) & <D_{k} \\
& <I\left(W ; V_{k} \mid U_{k}\right)-4 \delta H(W),
\end{aligned}
$$

and set the helper rate to be

$$
R_{k}=D_{k}+\epsilon_{\mathrm{h}}
$$

for some arbitrarily small $\epsilon_{\mathrm{h}}>0$. Notice that it is always possible to choose $D_{k}$ in (46) because (44) and (45) imply $H\left(X \mid Y_{k}\right)<I\left(W ; U_{k}, V_{k}\right)$ and $I\left(W ; V_{k} \mid U_{k}\right)>0$; we can choose $\delta$ arbitrarily small; and $H(W)$ is finite.

We construct a random codebook for helper $\mathrm{BS}(k)$ : The codebook is generated by applying the map $\phi_{k}$ symbol-bysymbol to each codeword $\mathbb{W}(m) \in \mathcal{C}_{W}$; that is,

$$
\mathcal{C}_{V_{k}}:=\bigcup_{m=1}^{M}\left\{\phi_{k}(\mathbb{W}(m))\right\},
$$

where

$$
\phi_{k}(\mathbb{W}(m))=\left(\phi_{k}\left(W_{1}(m)\right), \phi_{k}\left(W_{2}(m)\right), \ldots, \phi_{k}\left(W_{n}(m)\right)\right)
$$

is a slight abuse of notation.

Uniformly at random place each codeword in $\mathcal{C}_{V_{k}}$ into one of $\left\lceil 2^{n R_{k}}\right\rceil$ bins. Uniquely label each bin with an index from the set $\left\{1,2, \ldots,\left\lceil 2^{n R_{k}}\right\rceil\right\}$, and let $f_{k}(\vee)$ denote the bin index of the codeword $\vee \in \mathcal{C}_{V_{k}}$. Denote the set of all codewords in the $b$-th bin by $\mathcal{B}_{k}(b):=\left\{\vee \in \mathcal{C}_{V_{k}}: f_{k}(v)=b\right\}$ for $b \in\left\{1,2, \ldots,\left\lceil 2^{n R_{k}}\right\rceil\right\}$.

\section{B. Encoding and Decoding}

The list encoder and decoders operate as before, see Sections VI-C and VI-D Helper BS $(k)$ looks for $\mathbb{V}_{k}=\phi_{k}(\mathbb{W})$ in $\mathcal{C}_{V_{k}}$ and, if successful, sends the bin index $B=f_{k}\left(\mathbb{V}_{k}\right)$ to receiver $k$. If unsuccessful, the helper sends an index with an independent and uniform distribution.

The list decoder at receiver $k$ outputs $\mathcal{L}_{k}$, see [20, from which the receiver computes a new list of $V_{k}$-codewords:

$$
\mathcal{L}_{k}^{*}:=\left\{\vee \in \mathcal{C}_{V_{k}}: \exists \mathbb{X}(m) \in \mathcal{L}_{k} \text { with } \vee=\phi_{k}(\mathbb{W}(m))\right\} .
$$


If there is a unique codeword $\nabla^{\prime}$ in the intersection of the list $\mathcal{L}_{k}^{*}$ and the bin $\mathcal{B}_{k}(B)$, then receiver $k$ sets $\hat{\mathbb{V}}_{k}:=\nabla^{\prime}$. Otherwise, receiver $k$ generates $\hat{\mathbb{V}}_{k}$ iid $\sim P_{V_{k}}$.

Finally, receiver $k$ looks for a unique source codeword $\mathbb{X}\left(m^{\prime}\right) \in \mathcal{L}_{k}$ such that $\left(\mathbb{W}\left(m^{\prime}\right), \mathbb{U}_{k}, \hat{\mathbb{V}}_{k}\right) \in \mathcal{T}_{\epsilon}\left(P_{W, U_{k}, V_{k}}\right)$. If successful, the receiver outputs $\hat{\mathbb{K}}_{k}:=\mathbb{X}\left(m^{\prime}\right)$; otherwise, it selects a codeword $\mathfrak{X}(m)$ uniformly at random from $\mathcal{C}_{X}$.

\section{Error Analysis}

To bound the probability that receiver $k$ decodes in error, $\mathbb{P}\left[\hat{\mathbb{K}}_{k} \neq \mathbb{X}\right]$, it is useful to start with

$$
\mathbb{P}\left[\hat{\mathbb{X}}_{k} \neq \mathbb{X}\right] \leq \mathbb{P}\left[\hat{\mathbb{V}}_{k} \neq \mathbb{V}_{k}\right]+\mathbb{P}\left[\left\{\hat{\mathbb{V}}_{k}=\mathbb{V}_{k}\right\} \cap\left\{\hat{\mathbb{X}}_{k} \neq \mathbb{X}\right\}\right] .
$$

We may bound the probability that receiver $k$ incorrectly decodes $\mathbb{V}_{k}$ by conditioning on the list error event $\mathcal{E}$, defined in 21], and the encoder error $\left\{\mathbb{X} \notin \mathcal{C}_{X}\right\}$ as follows:

$$
\begin{aligned}
\mathbb{P}\left[\hat{\mathbb{V}}_{k} \neq \mathbb{V}_{k}\right] \leq \mathbb{P}[\mathcal{E}] & +\mathbb{P}\left[\mathbb{X} \notin \mathcal{C}_{X}\right] \\
& +\mathbb{P}\left[\hat{\mathbb{V}}_{k} \neq \mathbb{V}_{k} \mid \mathcal{E}^{c} \cap\left\{\mathbb{X} \in \mathcal{C}_{X}\right\}\right] .
\end{aligned}
$$

Upper bounds for $\mathbb{P}[\mathcal{E}]$ and $\mathbb{P}\left[\mathcal{K} \notin \mathcal{C}_{X}\right]$ are given in 23 and 24) respectively. Let us now rewrite the conditional probability in (49) using the law of total probability as

$$
\mathbb{P}\left[\hat{\mathbb{V}}_{k} \neq \mathbb{V}_{k} \mid \mathcal{E}^{c} \cap\left\{\mathbb{X} \in \mathcal{C}_{X}\right\}\right]
$$

$$
\begin{aligned}
& =\sum_{b=1}^{\left\lceil 2^{n R_{k}}\right\rceil} \mathbb{P}\left[\left\{f_{k}\left(\mathbb{V}_{k}\right)=b\right\} \cap\left\{\hat{\mathbb{V}}_{k} \neq \mathbb{V}_{k}\right\} \mid \mathcal{E}^{c} \cap\left\{\mathbb{X} \in \mathcal{C}_{X}\right\}\right] \\
& =\sum_{b=1}^{\left\lceil 2^{n R_{k}}\right\rceil} \mathbb{P}\left[f_{k}\left(\mathbb{V}_{k}\right)=b \mid \mathcal{E}^{c} \cap\left\{\mathbb{X} \in \mathcal{C}_{X}\right\}\right] \\
& \quad \cdot \mathbb{P}\left[\hat{\mathbb{V}}_{k} \neq \mathbb{V}_{k} \mid\left\{f_{k}\left(\mathbb{V}_{k}\right)=b\right\} \cap \mathcal{E}^{c} \cap\left\{\mathbb{X} \in \mathcal{C}_{X}\right\}\right] .
\end{aligned}
$$

We now fix a bin $b$ and derive

$$
\begin{aligned}
& \mathbb{P}\left[\hat{\mathbb{V}}_{k} \neq \mathbb{V}_{k} \mid\left\{f_{k}\left(\mathbb{V}_{k}\right)=b\right\} \cap \mathcal{E}^{c} \cap\left\{\mathbb{X} \in \mathcal{C}_{X}\right\}\right] \\
& \stackrel{\mathrm{a}}{=} \mathbb{P}\left[\bigcup_{\substack{\boldsymbol{v} \in \mathcal{L}_{k}^{*} \\
\nabla \neq \phi_{k}(\mathbb{W})}}\left\{f_{k}(\mathbb{v})=b\right\} \mid\left\{f_{k}\left(\mathbb{V}_{k}\right)=b\right\} \cap \mathcal{E}^{c} \cap\left\{\mathbb{X} \in \mathcal{C}_{X}\right\}\right] \\
& \stackrel{\mathrm{b}}{\leq} \sum_{\substack{\nabla \in \mathcal{L}_{k}^{*} \\
\nabla \neq \phi_{k}(\mathbb{W})}} \mathbb{P}\left[f_{k}(\mathbb{v})=b \mid\left\{f_{k}\left(\mathbb{V}_{k}\right)=b\right\} \cap \mathcal{E}^{c} \cap\left\{\mathbb{X} \in \mathcal{C}_{X}\right\}\right] \\
& \stackrel{\mathrm{c}}{=} \sum_{\substack{\nabla \in \mathcal{L}_{k}^{*} \\
\nabla \neq \phi_{k}(\mathbb{W})}} \frac{1}{\left\lceil 2^{n R_{k}}\right\rceil} \\
& \stackrel{\mathrm{d}}{\leq} 2^{-n\left(R_{k}-D_{k}\right)} \\
& \stackrel{\mathrm{f}}{=} 2^{-n \epsilon_{\mathrm{h}}} .
\end{aligned}
$$

$$
\begin{aligned}
& \mathbb{P}\left[\hat{\mathbb{K}} \neq \mathbb{X} \mid \mathcal{S}_{3}\right] \stackrel{\mathrm{a}}{=} \sum_{l:|l| \leq 2^{n D_{k}}} \mathbb{P}\left[\mathcal{L}_{k}=l \mid \mathcal{S}_{3}\right] \mathbb{P}\left[\hat{\mathbb{K}}_{k} \neq \mathbb{X} \mid \mathcal{S}_{3} \cap\left\{\mathcal{L}_{k}=l\right\}\right] \\
& \stackrel{\mathrm{b}}{=} \sum_{l:|l| \leq 2^{n D_{k}}} \mathbb{P}\left[\mathcal{L}_{k}=l \mid \mathcal{S}_{3}\right]\left(\sum _ { m = 1 } ^ { M } \mathbb { P } \left[\left\{\mathbb{X} \neq \mathbb{X}\left(m^{\prime}\right), \forall m^{\prime}<m\right\} \cap\{\mathbb{X}=\mathbb{X}(m)\} \mid\right.\right. \\
& \left.\left.\mathcal{S}_{3} \cap\left\{\mathcal{L}_{k}=l\right\}\right] \mathbb{P}\left[\hat{\mathbb{K}}_{k} \neq \mathbb{X} \mid \mathcal{S}_{3, m}\right]\right) \\
& \stackrel{\mathrm{c}}{=} \sum_{l:|l| \leq 2^{n D_{k}}} \mathbb{P}\left[\mathcal{L}_{k}=l \mid \mathcal{S}_{3}\right]\left(\sum _ { m = 1 } ^ { M } \mathbb { P } \left[\left\{\mathbb{X} \neq \mathbb{X}\left(m^{\prime}\right), \forall m^{\prime}<m\right\} \cap\{\mathbb{K}=\mathbb{X}(m)\} \mid\right.\right. \\
& \left.\left.\mathcal{S}_{3} \cap\left\{\mathcal{L}_{k}=l\right\}\right] \mathbb{P}\left[\bigcup_{\substack{\tilde{m} \in l \\
\tilde{m} \neq m}}\left\{\left(\mathbb{W}(\tilde{m}), \mathbb{U}_{k}, \hat{\mathbb{V}}_{k}\right) \in \mathcal{T}_{\delta}\right\} \mid \mathcal{S}_{3, m}\right]\right) \\
& \stackrel{\mathrm{d}}{\leq} \sum_{l:|l| \leq 2^{n D_{k}}} \mathbb{P}\left[\mathcal{L}_{k}=l \mid \mathcal{S}_{3}\right]\left(\sum _ { m = 1 } ^ { M } \mathbb { P } \left[\left\{\mathbb{X} \neq \mathbb{X}\left(m^{\prime}\right), \forall m^{\prime}<m\right\} \cap\{\mathbb{X}=\mathbb{X}(m)\} \mid\right.\right. \\
& \left.\left.\mathcal{S}_{3} \cap\left\{\mathcal{L}_{k}=l\right\}\right] \sum_{\substack{\tilde{m} \in l \\
m \neq m}} \mathbb{P}\left[\left(\mathbb{W}(\tilde{m}), \mathbb{U}_{k}, \hat{\mathbb{V}}_{k}\right) \in \mathcal{T}_{\delta} \mid \mathcal{S}_{3, m}\right]\right) \\
& \stackrel{\mathrm{e}}{\leq} \sum_{l:|l| \leq 2^{n D_{k}}} \mathbb{P}\left[\mathcal{L}_{k}=l \mid \mathcal{S}_{3}\right]\left(\sum _ { m = 1 } ^ { M } \mathbb { P } \left[\left\{\mathbb{X} \neq \mathbb{X}\left(m^{\prime}\right), \forall m^{\prime}<m\right\} \cap\{\mathbb{X}=\mathbb{X}(m)\} \mid\right.\right. \\
& \left.\left.\mathcal{S}_{3} \cap\left\{\mathcal{L}_{k}=l\right\}\right] \sum_{\substack{\tilde{m} \in l \\
m \neq m}} \gamma_{n} 2^{-n\left(I\left(W ; V_{k} \mid U_{k}\right)-4 \delta H(W)\right)}\right) \\
& \stackrel{\mathrm{f}}{\leq} \gamma_{n} 2^{n D_{k}} 2^{-n\left(I\left(W ; V_{k} \mid U_{k}\right)-4 \delta H(W)\right)}
\end{aligned}
$$




$$
\begin{aligned}
\mathbb{P}\left[\left(\mathbb{W}(\tilde{m}), \mathbb{U}_{k}, \hat{\mathbb{V}}_{k}\right) \in \mathcal{T}_{\delta} \mid \mathcal{S}_{3, m}\right] & \stackrel{\text { e.1 }}{=} \mathbb{P}\left[\left(\mathbb{W}(\tilde{m}), \mathbb{U}_{k}, \mathbb{V}_{k}\right) \in \mathcal{T}_{\delta} \mid\{\mathbb{W}=\mathbb{W}(m)\} \cap\left\{\left(\mathbb{W}(\tilde{m}), \mathbb{U}_{k}\right) \in \mathcal{T}_{\delta}\right\} \cap\left\{\left(\mathbb{W}, \mathbb{U}_{k}, \mathbb{V}_{k}\right) \in \mathcal{T}_{\delta_{1}}\right\}\right] \\
& \stackrel{\text { e.2 }}{\leq} \frac{\mathbb{P}\left[\left(\mathbb{W}(\tilde{m}), \mathbb{U}_{k}, \mathbb{V}_{k}\right) \in \mathcal{T}_{\delta} \mid\{\mathbb{W}=\mathbb{W}(m)\} \cap\left\{\left(\mathbb{W}, \mathbb{U}_{k}, \mathbb{V}_{k}\right) \in \mathcal{T}_{\delta_{1}}\right\}\right]}{\mathbb{P}\left[\left(\mathbb{W}(\tilde{m}), \mathbb{U}_{k}\right) \in \mathcal{T}_{\delta} \mid\{\mathbb{W}=\mathbb{W}(m)\} \cap\left\{\left(\mathbb{W}, \mathbb{U}_{k}, \mathbb{V}_{k}\right) \in \mathcal{T}_{\delta_{1}}\right\}\right]} \\
& \stackrel{\text { e.3 }}{\leq} \gamma_{n} \frac{2^{-n\left(I\left(W ; U_{k}, V_{k}\right)-2 \delta H(W)\right)}}{2^{-n\left(I\left(W ; U_{k}\right)+2 \delta H(W)\right)}} .
\end{aligned}
$$

$$
\sum_{l:|l| \leq 2^{n D_{k}}} \mathbb{P}\left[\mathcal{L}_{k}=l \mid \mathcal{S}_{3}\right]\left(\sum_{m=1}^{M} \mathbb{P}\left[\left\{\mathbb{X} \neq \mathbb{X}\left(m^{\prime}\right), \forall m^{\prime}<m\right\} \cap\{\mathbb{X}=\mathbb{X}(m)\} \mid \mathcal{S}_{3} \cap\left\{\mathcal{L}_{k}=l\right\}\right]\right)=1 .
$$

Notes on 51):

a. receiver $k$ decodes $\mathbb{V}_{k}$ in error if and only if there is another $\vee \in \mathcal{L}_{k}^{*}$ assigned to the same bin as the correct $\nabla$-codeword;

b. the union bound;

c. the codewords in $\mathcal{C}_{V_{k}}$ are thrown uniformly at random into $\left\lceil 2^{n R_{k}}\right\rceil$ bins;

d. $\left|\mathcal{L}_{V_{k}}\right| \leq 2^{n D_{k}}$, since we condition on $\mathcal{E}^{c}$; and

f. substitute the choice of helper rate $R_{k}$ in (47).

The right-hand side of (51) is independent of $b$, so 50 gives

$$
\mathbb{P}\left[\hat{\mathbb{V}}_{k} \neq \mathbb{V}_{k} \mid \mathcal{E}^{c} \cap\left\{\mathbb{X} \in \mathcal{C}_{X}\right\}\right] \leq 2^{-n \epsilon_{\mathrm{h}}} .
$$

Combining 49, 23, 24 and 52 gives

$$
\mathbb{P}\left[\hat{\mathbb{V}}_{k} \neq \mathbb{V}_{k}\right] \leq b_{5} 2^{-a_{5} n},
$$

for some finite $a_{5}, b_{5}>0$.

We now turn to the rightmost probability in (48). We have

$$
\mathbb{P}\left[\left\{\hat{\mathbb{V}}_{k}=\mathbb{V}_{k}\right\} \cap\left\{\hat{\mathbb{K}}_{k} \neq \mathbb{X}\right\}\right] \leq \mathbb{P}\left[\mathcal{S}_{3}^{c}\right]+\mathbb{P}\left[\hat{\mathbb{K}}_{k} \neq \mathbb{X} \mid \mathcal{S}_{3}\right],
$$

where

$$
\begin{aligned}
\mathcal{S}_{3}:= & \left\{\mathbb{X} \in \mathcal{C}_{X}\right\} \cap\left\{\left(\mathbb{K}, \mathbb{Y}_{k}\right) \in \mathcal{T}_{\epsilon_{1}}\left(P_{X, Y_{k}}\right)\right\} \cap\left\{\hat{\mathbb{V}}_{k}=\mathbb{V}_{k}\right\} \\
& \cap\left\{\left(\mathbb{W}, \mathbb{U}_{k}, \mathbb{V}_{k}\right) \in \mathcal{T}_{\delta_{1}}\left(P_{W, U_{k}, V_{k}}\right)\right\} \cap\left\{\left|\mathcal{L}_{k}\right| \leq 2^{n D_{k}}\right\} .
\end{aligned}
$$

An upper bound on the probability $\mathbb{P}\left[\mathcal{S}_{3}^{c}\right]$ in 54 follows easily from previous bounds:

$$
\begin{aligned}
\mathbb{P}\left[\mathcal{S}_{3}^{c}\right] \leq \mathbb{P}\left[\mathbb{X} \notin \mathcal{C}_{X}\right]+\mathbb{P}\left[\left(\mathbb{X}, \mathbb{Y}_{k}\right) \notin \mathcal{T}_{\epsilon_{1}}\right]+\mathbb{P}\left[\hat{\mathbb{V}}_{k} \neq \mathbb{V}_{k}\right] \\
\quad+\mathbb{P}\left[\left(\mathbb{W}, \mathbb{U}_{k}, \mathbb{V}_{k}\right) \notin \mathcal{T}_{\delta_{1}}\right]+\mathbb{P}\left[\left|\mathcal{L}_{k}\right|>2^{n D_{k}}\right] \\
\\
\quad * b_{6} 2^{-a_{6} n}
\end{aligned}
$$

where $(*)$ holds for some finite $a_{6}, b_{6}>0$ by 24, 41, (53) and Lemma 4.

The rightmost probability in (54) is bounded by

$$
\mathbb{P}\left[\hat{\mathbb{V}}_{k} \neq \mathbb{X} \mid \mathcal{S}_{3}\right] \leq \gamma_{n} 2^{-n\left(I\left(W ; V_{k} \mid U_{k}\right)-D_{k}+4 \delta H(W)\right)},
$$

where

$$
\gamma_{n}:=\frac{\exp \left(2 n\left(1-\delta_{1}\right) \frac{\left(\delta-\delta_{1}\right)^{2}}{1+\delta_{1}} \mu_{W, U_{k}}^{2}\right)}{\exp \left(2 n\left(1-\delta_{1}\right) \frac{\left(\delta-\delta_{1}\right)^{2}}{1+\delta_{1}} \mu_{W, U_{k}}^{2}\right)-2|\mathcal{W}|\left|\mathcal{U}_{k}\right|}
$$

The steps leading to 56 are shown above in 57. Notes:

a. Write $\mathbb{P}\left[\hat{\mathbb{K}}_{k} \neq \mathbb{X} \mid \mathcal{S}_{3}\right]$ as an expectation over all possible realisations of decoder $k$ 's list $\mathcal{L}_{k}$. We note that $\left|\mathcal{L}_{k}\right| \leq$ $2^{n D_{k}}$ with probability one, after conditioning on $\mathcal{S}_{3}$.

b. Let

$$
\begin{aligned}
\mathcal{S}_{3, m}:=\left\{\mathbb{X} \neq \mathbb{X}\left(m^{\prime}\right), \forall m^{\prime}<m\right\} & \cap\{\mathbb{X}=\mathbb{X}(m)\} \\
& \cap \mathcal{S}_{3} \cap\{\mathcal{L}=l\} .
\end{aligned}
$$

and note that $\mathbb{X} \in \mathcal{C}_{X}$, after conditioning on $\mathcal{S}_{3}$.

c. An error may only occur if there is some other index $\tilde{m} \neq m$ such that $\mathbb{X}(\tilde{m}) \in \mathcal{L}_{k}$ and $\left(\mathbb{W}(\tilde{m}), \mathbb{U}_{k}, \hat{\mathbb{V}}_{k}\right)$ is jointly typical. Here we note that after conditioning on $\mathcal{S}_{3, m}$ the following holds with probability one: the source $\mathbb{X}$ equals the $m$-th codeword $\mathbb{X}(m)$ in the source codebook $\mathcal{C}_{X}$; the $m$-th channel codeword is transmitted $\mathbb{W}=\mathbb{W}(m)$; the source and side information $\left(\mathbb{X}, \mathbb{Y}_{k}\right)$ are $\epsilon$-jointly typical; $\left(\mathbb{W}, \mathbb{U}_{k}, \mathbb{V}_{k}\right)$ are $\delta$-jointly typical; and $\hat{\mathbb{V}}=\mathbb{V}$. We have also abbreviated $\mathcal{T}_{\epsilon_{1}}\left(P_{X, Y_{k}}\right)$ and $\mathcal{T}_{\delta_{1}}\left(P_{W, U_{k}, V_{k}}\right)$ as $\mathcal{T}_{\epsilon_{1}}$ and $\mathcal{T}_{\delta_{1}}$ respectively.

d. Apply the union bound.

e. The rightmost probability in step (d) is bounded by

$$
\begin{aligned}
\mathbb{P}\left[\left(\mathbb{W}(\tilde{m}), \mathbb{U}_{k}, \hat{\mathbb{V}}_{k}\right)\right. & \left.\in \mathcal{T}_{\delta} \mid \mathcal{S}_{3, m}\right] \\
& \leq \gamma_{n} 2^{-n\left(I\left(W ; V_{k} \mid U_{k}\right)-4 \delta H(W)\right)}
\end{aligned}
$$

The steps leading to this bound are shown above in (58). (See below for detailed notes on each step in (58).)

f. For each list $l$ that satisfies $|l| \leq 2^{n D_{k}}$,

$$
\begin{aligned}
\sum_{\substack{\tilde{m} \in l \\
\tilde{m} \neq m}} \gamma_{n} 2^{-n\left(I\left(W ; V_{k} \mid U_{k}\right)-4 \delta H(W)\right)} & \\
& \leq \gamma_{n} 2^{n D_{k}} 2^{-n\left(I\left(W ; V_{k} \mid U_{k}\right)-4 \delta H(W)\right)} .
\end{aligned}
$$

Step (f) now follows from (59) above.

Notes for (58):

e.1. This step follows from the independence of the source and channel codebooks, the independence of codewords within each codebook, conditioning on $\mathcal{S}_{3, m}$ and $\left\{\mathcal{L}_{k}=\right.$ l) being equivalent to

$$
\left(\mathbb{X}\left(m^{\prime}\right), \mathbb{Y}_{k}\right) \in \mathcal{T}_{\epsilon} \text { and }\left(\mathbb{W}\left(m^{\prime}\right), \mathbb{U}_{k}\right) \in \mathcal{T}_{\delta}, \forall m^{\prime} \in l,
$$


and

$$
\left(\mathcal{X}\left(m^{\prime}\right), \mathbb{Y}_{k}\right) \notin \mathcal{T}_{\epsilon} \text { or }\left(\mathbb{W}\left(m^{\prime}\right), \mathbb{U}_{k}\right) \notin \mathcal{T}_{\delta}, \forall m^{\prime} \notin l .
$$

e.2. Bayes' rule.

e.3. Apply Lemma 5 to (e.2).

Thus,

$$
\mathbb{P}\left[\hat{\mathbb{K}}_{k} \neq \mathbb{X} \mid \mathcal{S}_{3}\right] \leq b_{7} 2^{-a_{7} n},
$$

for some $b_{7}>0$ and $a_{7}:=I\left(W ; V_{k} \mid U_{k}\right)-D_{k}-4 \delta H(W)$, where $a_{7}>0$ by (46). Whenever (44) and (45) both hold, the achievability achievability of Theorem 1 follows from (48) and (53), (54), (55), and (60).

To complete the achievability proof of Theorem 1 , we need only relax the assumption (45) and suppose that $H\left(X \mid Y_{k}\right)<$ $I\left(W ; U_{k}\right)$ for one or more receivers $k$. Such receivers do not require a positive helper rate or list exponent (i.e., we can set $R_{k}=0$ and $D_{k}=0$ ), and we can instead impose unique decoding. Indeed, the error analysis in [8. Sec. IV] shows that the probability of error $\mathbb{P}\left[\hat{\mathbb{K}}_{k} \neq \mathbb{X}\right]$ at such receivers decays exponentially in $n$. (The error analysis in [8, Sec. IV] is valid because we use the same random source and channel codebooks.)

\section{Proof of Theorem 2 - Converse}

Fix $\epsilon>0$. Consider any $\left(n_{\mathrm{s}}, n_{\mathrm{c}}, R_{1}, R_{2}, \ldots, R_{K}\right)$-code with $\mathbb{P}\left[\hat{\mathbb{K}}_{k} \neq \mathbb{X}\right] \leq \epsilon$ for all $k$. Following the now familiar path of defining $\left(\tilde{W}, \tilde{U}_{1}, \tilde{U}_{2}, \ldots, \tilde{U}_{K}\right) \sim P_{\tilde{W}}(\cdot) T(\cdot \mid \cdot)$, with the timeshared pmf $P_{\tilde{W}}$ given in (15), we have

$$
\begin{aligned}
n_{\mathrm{c}} I\left(\tilde{W} ; \tilde{U}_{k}\right) & \stackrel{\mathrm{a}}{\geq} I\left(\mathbb{W} ; \mathbb{U}_{k}\right) \stackrel{\mathrm{b}}{=} I\left(\mathbb{X}, \mathbb{\mho}_{k}, M_{k}, \mathbb{W} ; \mathbb{U}_{k}\right) \\
& \geq H\left(\mathbb{X} \mid M_{k}, \mathbb{\mho}_{k}\right)-H\left(\mathbb{X} \mid M_{k}, \mathbb{Y}_{k}, \mathbb{U}_{k}\right) \\
& \geq \sum_{i=1}^{n_{\mathrm{s}}} H\left(X_{i} \mid M_{k}, \mathbb{\mho}_{k}, X_{1}^{i-1}\right)-n_{\mathrm{s}} \varepsilon\left(n_{\mathrm{s}}\right) \\
& \geq \sum_{i=1}^{n_{\mathrm{s}}} H\left(X_{i} \mid M_{k}, \mathbb{\mho}_{k}, X_{1}^{i-1}, V_{k, 1}^{i-1}\right)-n_{\mathrm{s}} \varepsilon\left(n_{\mathrm{s}}\right) \\
& \stackrel{\mathrm{e}}{=} \sum_{i=1}^{n_{\mathrm{s}}} H\left(X_{i} \mid M_{k}, \mathbb{Y}_{k}, V_{k, 1}^{i-1}\right)-n_{\mathrm{s}} \varepsilon\left(n_{\mathrm{s}}\right) \\
& \stackrel{\mathrm{f}}{=} \sum_{i=1}^{n_{\mathrm{s}}} H\left(X_{i} \mid A_{k, i}, Y_{k, i}\right)-n_{\mathrm{s}} \varepsilon\left(n_{\mathrm{s}}\right)
\end{aligned}
$$

Notes:

a. Jensen's inequality;

b. $\left(\mathbb{X}, \mathbb{Y}_{k}, M_{k}\right) \leftrightarrow \mathbb{W} \leftrightarrow \mathbb{U}_{k}$ forms a Markov chain;

c. Fano's inequality, where $\varepsilon(n) \rightarrow 0$, and the shorthand notation $X_{1}^{i-1}=\left(X_{1}, X_{2}, \ldots, X_{i-1}\right)$;

d. conditioning reduces entropy and the notation $V_{k, 1}^{i-1}=$ $\left(V_{k, 1}, V_{k, 2}, \ldots, V_{k, i-1}\right)$;

e. $X_{i} \leftrightarrow\left(M_{k}, V_{k, 1}^{i-1}, \Upsilon_{k}\right) \leftrightarrow X_{1}^{i-1}$ forms a Markov chain (see below for details); and

f. substitutes $A_{k, i}:=\left(M_{k}, Y_{k, 1}^{i-1}, Y_{k, i+1}^{n}, V_{k, 1}^{i-1}\right)$.

To see that $X_{i} \leftrightarrow\left(M_{k}, V_{k, 1}^{i-1}, \Upsilon_{k}\right) \leftrightarrow X_{1}^{i-1}$ forms a Markov chain in step (e) above, we first notice that

$$
\left(X_{i}, M_{k}, Y_{k, i}^{n}\right) \leftrightarrow V_{k, 1}^{i-1} \leftrightarrow\left(X_{1}^{i-1}, Y_{1}^{i-1}\right)
$$

forms a Markov chain because the source and side information are memoryless and $M_{k}$ is a function only of $\mathbb{V}_{k}$. The chain [62 implies $X_{i} \leftrightarrow\left(M_{k}, V_{k, 1}^{i-1}, Y_{k, i}^{n}\right) \leftrightarrow\left(X_{1}^{i-1}, Y_{1}^{i-1}\right)$, which, in turn, implies $X_{i} \leftrightarrow\left(M_{k}, V_{k, 1}^{i-1}, \mho_{k}\right) \leftrightarrow X_{1}^{i-1}$.

The bound for helper rate $R_{k}$ follows a similar argument to that of [23, Sec. 15.8]. Specifically,

$$
\begin{aligned}
n_{\mathrm{s}} R_{k} & \geq H\left(M_{k}\right) \geq I\left(\mathbb{V}_{k} ; M_{k} \mid \mathbb{Y}_{k}\right) \\
& =\sum_{i=1}^{n_{\mathrm{s}}} I\left(V_{k, i} ; M_{k} \mid \mathbb{Y}_{k}, V_{k, 1}^{i-1}\right) \\
& \stackrel{\mathrm{a}}{=} \sum_{i=1}^{n_{\mathrm{s}}} I\left(V_{k, i} ; M_{k}, Y_{k, 1}^{i-1}, Y_{k, i+1}^{n}, V_{k, 1}^{i-1} \mid Y_{k, i}\right) \\
& \stackrel{\mathrm{b}}{=} \sum_{i=1}^{n_{\mathrm{s}}} I\left(V_{k, i} ; A_{k, i} \mid Y_{k, i}\right),
\end{aligned}
$$

where step (a) follows because the source is memoryless and (b) substitutes $A_{k, i}$.

The source and the side information are iid and $M_{k}$ is only a function of $\mathbb{V}_{k}$, so

$$
\left(X_{i}, Y_{i}\right) \leftrightarrow V_{k, i} \leftrightarrow\left(M_{k}, \mathbb{V}_{k}, Y_{k, 1}^{i-1}, Y_{k, i+1}^{n}\right) .
$$

The Markov chain (63) implies $\left(X_{i}, Y_{i}\right) \leftrightarrow V_{k, i} \leftrightarrow A_{k, i}$, and the converse follows from standard timesharing arguments, e.g. see [23, p. 578].

\section{Proof of Theorem 2 - Achievability}

The proof combes the list decoder of Section VI] with a 'helper' source code at $\mathrm{BS}(k)$.

\section{A. Code Construction}

Fix a pmf $P_{W}$ on $\mathcal{W}$ and auxiliary random variables $\left(A_{1}\right.$, $\left.A_{2}, \ldots, A_{K}\right)$ satisfying $A_{k} \leftrightarrow V_{k} \leftrightarrow\left(X, Y_{k}\right)$. Let us assume that

$$
H\left(X \mid A_{k}, Y_{k}\right)<I\left(W ; U_{k}\right) \quad \forall k
$$

and

$$
R_{k}>I\left(A_{k} ; V_{k} \mid Y_{k}\right) \quad \forall k .
$$

As in Section VIII (the achievability proof Theorem 1), let us also assume that (45) holds so that every receiver requires a positive helper rate.

Fix constants $\epsilon, \epsilon_{1}, \delta$ and $\delta_{1}$ satisfying (18), and choose $0<\epsilon_{1}<\epsilon_{\mathrm{h} 1}<\epsilon_{\mathrm{h}}<\mu_{A_{k}, X, Y_{k}}$. Generate a random list code, as described in Section VI, with the parameters described above, and let $\mathcal{C}_{X}$ and $\mathcal{C}_{W}$ denote the source and channel codebooks respectively. Fix the list exponents to be

$$
D_{k}=H\left(X \mid Y_{k}\right)-I\left(W ; U_{k}\right)+\rho, \quad \forall k,
$$

for any

$$
\rho>3 \epsilon H(X)+2 \delta H(W) .
$$

Let $P_{A_{k}}$ denote the marginal distribution of $A_{k}$. Randomly generate a source codebook for $\mathrm{BS}(k)$, with codewords of length $n$, by selecting symbols from $\mathcal{A}_{k}$ iid $\sim P_{A_{k}}$ :

$\mathcal{C}_{A_{k}}:=\left\{A_{k}\left(j, j^{\prime}\right)=\left(A_{k, 1}\left(j, j^{\prime}\right), A_{k, 2}\left(j, j^{\prime}\right), \ldots, A_{k, n}\left(j, j^{\prime}\right)\right)\right\}$ where we call $j$ the bin index and $j=1,2, \ldots,\left\lfloor 2^{n R_{k}}\right\rfloor$ and $j^{\prime}=1,2, \ldots,\left\lfloor 2^{n\left(I\left(A_{k} ; Y_{k}\right)-\epsilon_{\mathrm{h} 1}\right)}\right\rfloor$. 


\section{B. Encoding and Decoding}

The list encoder and decoders operate as before, see Sections VI-C and VI-D. Helper BS $(k)$ searches through the $A_{k}$ codebook $\mathcal{C}_{A_{k}}$ for a pair $\left(J, J^{\prime}\right)$ such that $\left(\mathbb{A}_{k}\left(J, J^{\prime}\right), \mathbb{V}_{k}\right) \in$ $\mathcal{T}_{\epsilon_{\mathrm{h} 1}}$. If successful, $\mathrm{BS}(k)$ sends the smallest such bin index $J$ to receiver $k$. If unsuccessful, the helper sends an index $J$ with an independent and uniform distribution over all possible bin indices.

Receiver $k$ first attempts to decode $\mathbb{A}_{k}\left(J, J^{\prime}\right)$ by looking for a unique $\hat{J}$ in the $J$-th bin such that $\left(\mathbb{A}_{k}(J, \hat{J}), \Upsilon_{k}\right) \in \mathcal{T}_{\epsilon_{\mathrm{h} 1}}$. If successful, receiver $k$ sets $\hat{A}_{k}=\mathbb{A}_{k}(J, \hat{J})$. Otherwise, it randomly selects $\hat{A}_{k}$ iid $\sim P_{A_{k}}$.

The list decoder at receiver $k$ outputs $\mathcal{L}_{k}$, see 20. Receiver $k$ looks for a unique $\mathbb{X}\left(m^{\prime}\right) \in \mathcal{L}_{k}$ such that $\left(\mathbb{X}\left(m^{\prime}\right), \mho_{k}, \hat{A}_{k}\right) \in \mathcal{T}_{\epsilon}$. If successful, receiver $k$ outputs $\hat{\mathbb{K}}_{k}:=$ $\mathcal{X}\left(m^{\prime}\right)$. Otherwise, it randomly generates $\hat{\mathbb{K}}_{k}$ using $P_{X}$.

\section{Error Analysis}

We first bound the probability of error at receiver $k$ by

$$
\mathbb{P}\left[\hat{\mathbb{K}}_{k} \neq \mathbb{X}\right] \leq \mathbb{P}\left[\mathcal{S}_{4}^{c}\right]+\mathbb{P}\left[\hat{\mathbb{K}}_{k} \neq \mathbb{X} \mid \mathcal{S}_{4}\right],
$$

where

$$
\begin{aligned}
\mathcal{S}_{4}:=\left\{\mathbb{X} \in \mathcal{C}_{X}\right\} & \cap\left\{\hat{\mathbb{A}}_{k}=\mathbb{A}_{k}\right\} \cap\left\{\left|\mathcal{L}_{k}\right| \leq 2^{n D_{k}}\right\} \\
& \cap\left\{\left(\mathbb{X}, \mathbb{Y}_{k}, \mathbb{A}_{k}\right) \in \mathcal{T}_{\epsilon_{1}}\right\} \cap\left\{\left(\mathbb{W}, \mathbb{U}_{k}\right) \in \mathcal{T}_{\delta_{1}}\right\},
\end{aligned}
$$

and we have abbreviated the typical sets $\mathcal{T}_{\epsilon_{1}}\left(P_{X, Y_{k}, A_{k}}\right)$ and $\mathcal{T}_{\delta_{1}}\left(P_{W, U_{k}}\right)$ as $\mathcal{T}_{\epsilon_{1}}$ and $\mathcal{T}_{\delta_{1}}$, respectively.

We have

$$
\mathbb{P}\left[\mathcal{S}_{4}^{c}\right] \leq b_{7} 2^{-a_{7} n},
$$

for some finite $a_{7}, b_{7}>0$. To see (67), apply the union bound to $\mathbb{P}\left[\mathcal{S}_{4}^{c}\right]$; use $(25)$ to bound $\mathbb{P}\left[\mathbb{K} \notin \mathcal{C}_{X}\right]$; use $[40$, (41) and (66) to bound $\mathbb{P}\left[\left|\mathcal{L}_{k}\right|>2^{n D_{k}}\right]$; and use Lemmas 4 and 5 to bound $\mathbb{P}\left[\left(\mathbb{W}, \mathbb{U}_{k}\right) \notin \mathcal{T}_{\delta_{1}}^{n}\right]$. The final two probabilities, $\mathbb{P}\left[\hat{A}_{k} \neq \mathbb{A}\right]$ and $\mathbb{P}\left[\left(\mathcal{X}, \mathbb{Y}_{k}, \mathbb{A}_{k}\right) \notin \mathcal{T}_{\epsilon_{1}}^{n}\right]$, also tend to zero exponentially in $n$ by Lemmas 4 and 5 , see, for example, Kramer's achievabiltiy proof of the Wyner-Ziv theorem [33. Sec. 5.3]. Finally, the conditional probability $\mathbb{P}\left[\hat{\mathbb{K}}_{k} \neq \mathrm{X} \mid \hat{\mathcal{S}}_{4}\right]$ tends to zero exponentially in $n$, as shown below in 68.

Notes for 68):

a. Write $\mathbb{P}\left[\hat{\mathbb{K}}_{k} \neq \mathbb{X} \mid \mathcal{S}_{4}\right]$ as an expectation over all possible realisations of decoder $k$ 's list $\mathcal{L}_{k}$. Here we note that $\left|\mathcal{L}_{k}\right| \leq 2^{n D_{k}}$ with probability one, after conditioning on $\mathcal{S}_{4}$.

b. Write the second conditional probability in step (a) as an expectation over all possible encodings of $\mathfrak{X}$. Here we note that $\mathbb{X} \in \mathcal{C}_{X}$ with probability one, after conditioning on $\mathcal{S}_{4}$.

c. In the rightmost conditional probability in step (b), the error event $\left\{\hat{X}_{k} \neq \mathbb{X}\right\}$ is equivalent to the following: There exists an index $\tilde{m}$ in decoder $k$ 's list $\mathcal{L}_{k}$, which is different to the correct index $m$ and such that $\left(\mathbb{X}(\tilde{m}), \mathbb{Y}_{k}, \hat{A}_{k}\right)$ is jointly typical. Here we note that the correct index $m$ is in decoder $k$ 's list with probability one, after conditioning on $\mathcal{S}_{4}$. We have also slightly abused notation and written the union over all indices $m^{\prime} \in \mathcal{L}_{k}$, but $\mathcal{L}_{k}$ is a list of source codewords, see 20 . d. Apply the union bound to (c).

e. If the index $\tilde{m}$ is smaller than $m, \tilde{m}<m$, then the rightmost conditional probability in step (d) is bounded from above by (69), which is given below. It can also be shown that 69) holds for indices $\tilde{m}>m$. To see this note that $\gamma_{n}>1$ and the righthand side of step (e.1) in 69. simplifies to

$$
\begin{aligned}
\mathbb{P}\left[\left(\mathbb{X}(\tilde{m}), \mathbb{Y}_{k}, \mathbb{A}_{k}\right) \in \mathcal{T}_{\epsilon} \mid\left\{\left(\mathbb{X}(\tilde{m}), \mathbb{Y}_{k}\right) \in \mathcal{T}_{\epsilon}\right\}\right. \\
\left.\cap\left\{\left(\mathbb{X}, \mathbb{Y}_{k}, \mathbb{A}_{k}\right) \in \mathcal{T}_{\epsilon_{1}}\right\}\right] .
\end{aligned}
$$

The bound then follows from Lemma 5 .

f. Substitute $D_{k}=H\left(X \mid Y_{k}\right)-I\left(W ; U_{k}\right)+\rho$.

Notes for 69):

e.1. The first step is a consequence of the independence of the source and channel codebooks, the independence of codewords within each codebook, and $\left\{\mathcal{L}_{k}=l\right\}$ is equivalent to

$$
\left(\mathbb{X}\left(m^{\prime}\right), \Upsilon_{k}\right) \in \mathcal{T}_{\epsilon} \text { and }\left(\mathbb{W}\left(m^{\prime}\right), \mathbb{U}_{k}\right) \in \mathcal{T}_{\delta}, \quad \forall m^{\prime} \in l,
$$

and

$$
\left(\mathbb{X}\left(m^{\prime}\right), \mathbb{\mho}_{k}\right) \notin \mathcal{T}_{\epsilon} \text { or }\left(\mathbb{W}\left(m^{\prime}\right), \mathbb{U}_{k}\right) \notin \mathcal{T}_{\delta}, \quad \forall m^{\prime} \notin l .
$$

e.2. Apply Bayes' law twice and use the upper bound

$$
\begin{aligned}
\mathbb{P}[\{\mathbb{X} \neq \mathbb{X}(\tilde{m})\} & \cap\left\{\left(\mathbb{X}(\tilde{m}), \mathbb{Y}_{k}\right) \in \mathcal{T}_{\epsilon}\right\} \mid\{(\mathbb{X}(\tilde{m}), \\
\left.\mathbb{Y}_{k}, \mathbb{A}_{k}\right) & \left.\left.\in \mathcal{T}_{\epsilon}\right\} \cap\left\{\left(\mathbb{X}, \mathbb{Y}_{k}, \mathbb{A}_{k}\right) \in \mathcal{T}_{\epsilon_{1}}\right\}\right] \leq 1
\end{aligned}
$$

e.3. Use Lemmas 4 and 5 to lower bound

$$
\begin{aligned}
1-\mathbb{P}\left[\mathbb{X}=\mathbb{X}(\tilde{m}) \mid\left\{\left(\mathbb{X}(\tilde{m}), \mathbb{Y}_{k}\right) \in \mathcal{T}_{\epsilon}\right\}\right. & \\
& \left.\cap\left\{\left(\mathbb{X}, \mathbb{Y}_{k}, \mathbb{A}_{k}\right) \in \mathcal{T}_{\epsilon_{1}}\right\}\right] .
\end{aligned}
$$

Use Lemma 5 to bound the numerator and denominator of the rightmost term in step (e.2).

e.4. Set

$$
\gamma_{n}:=\frac{1}{1-\frac{1}{1-\zeta_{n}} 2^{-n\left(H\left(X \mid Y_{k}\right)-3 \epsilon H(X)\right)}}
$$

where

$$
\zeta_{n}:=2|\mathcal{X}|\left|\mathcal{Y}_{k}\right| \exp \left(-2 n\left(1-\epsilon_{1}\right) \frac{\left(\epsilon-\epsilon_{1}\right)^{2}}{1+\epsilon_{1}} \mu_{X, Y_{k}}^{2}\right)
$$

We notice that $\gamma_{n} \rightarrow 1$ from above whenever $3 \epsilon H(X)<$ $H\left(X \mid Y_{k}\right)$ and $\epsilon>\epsilon_{1}$.

The proof now follows from (69), because $H\left(X \mid A_{k}, Y_{k}\right)<$ $I\left(W ; U_{k}\right)$, we can choose $\epsilon$ and $\rho$ arbitrarily small, and $H(X)$ is finite.

\section{ACKNOWLEDGEMENTS}

The authors would like to thank Gerhard Kramer, the associate editor and the anonymous reviewers for their thoughtful comments on the paper. 


$$
\begin{aligned}
& \mathbb{P}\left[\hat{\mathbb{X}}_{k} \neq \mathbb{X} \mid \mathcal{S}_{4}\right] \stackrel{\mathrm{a}}{=} \sum_{l:|l| \leq 2^{n D_{k}}} \mathbb{P}\left[\mathcal{L}_{k}=l \mid \mathcal{S}_{4}\right] \mathbb{P}\left[\hat{\mathbb{K}}_{k} \neq \mathbb{X} \mid \mathcal{S}_{4} \cap\{\mathcal{L}=l\}\right] \\
& \stackrel{\mathrm{b}}{=} \sum_{l:|l| \leq 2^{n D_{k}}} \mathbb{P}\left[\mathcal{L}_{k}=l \mid \mathcal{S}_{4}\right]\left(\sum_{m=1}^{M} \mathbb{P}\left[\left\{\mathbb{X} \neq \mathbb{X}\left(m^{\prime}\right), \forall m^{\prime}<m\right\} \cap\{\mathbb{X}=\mathbb{X}(m)\} \mid \mathcal{S}_{4} \cap\left\{\mathcal{L}_{k}=l\right\}\right]\right. \\
& \left.\cdot \mathbb{P}\left[\hat{\mathbb{X}}_{k} \neq \mathbb{X} \mid\left\{\mathbb{X} \neq \mathbb{X}\left(m^{\prime}\right), \forall m^{\prime}<m\right\} \cap\{\mathbb{X}=\mathbb{X}(m)\} \cap \mathcal{S}_{4} \cap\left\{\mathcal{L}_{k}=l\right\}\right]\right) \\
& \stackrel{\mathrm{c}}{=} \sum_{l:|l| \leq 2^{n D_{k}}} \mathbb{P}\left[\mathcal{L}_{k}=l \mid \mathcal{S}_{4}\right]\left(\sum_{m=1}^{M} \mathbb{P}\left[\left\{\mathbb{X} \neq \mathbb{X}\left(m^{\prime}\right), \forall m^{\prime}<m\right\} \cap\{\mathbb{X}=\mathbb{X}(m)\} \mid \mathcal{S}_{4} \cap\left\{\mathcal{L}_{k}=l\right\}\right]\right. \\
& \left.\cdot \mathbb{P}\left[\bigcup_{\substack{\tilde{m} \in l \\
\tilde{m} \neq m}}\left\{\left(\mathbb{X}(\tilde{m}), \mathbb{Y}_{k}, \hat{\mathbb{A}}_{k}\right) \in \mathcal{T}_{\epsilon}\right\} \mid\left\{\mathbb{X} \neq \mathbb{X}\left(m^{\prime}\right), \forall m^{\prime}<m\right\} \cap\{\mathbb{X}=\mathbb{X}(m)\} \cap \mathcal{S}_{4} \cap\left\{\mathcal{L}_{k}=l\right\}\right]\right) \\
& \stackrel{\mathrm{d}}{\leq} \sum_{l:|l| \leq 2^{n D_{k}}} \mathbb{P}\left[\mathcal{L}_{k}=l \mid \mathcal{S}_{4}\right]\left(\sum_{m=1}^{M} \mathbb{P}\left[\left\{\mathbb{X} \neq \mathbb{X}\left(m^{\prime}\right), \forall m^{\prime}<m\right\} \cap\{\mathbb{X}=\mathbb{X}(m)\} \mid \mathcal{S}_{4} \cap\left\{\mathcal{L}_{k}=l\right\}\right]\right. \\
& \left.\cdot\left(\sum_{\substack{\tilde{m} \in l \\
\tilde{m} \neq m}} \mathbb{P}\left[\left(\mathbb{X}(\tilde{m}), \mathbb{Y}_{k}, \hat{A}_{k}\right) \in \mathcal{T}_{\epsilon} \mid\left\{\mathbb{X} \neq \mathbb{X}\left(m^{\prime}\right), \forall m^{\prime}<m\right\} \cap\{\mathbb{X}=\mathbb{X}(m)\} \cap \mathcal{S}_{4} \cap\left\{\mathcal{L}_{k}=l\right\}\right]\right)\right) \\
& \stackrel{\mathrm{e}}{\leq} \gamma_{n} 2^{-n\left(I\left(X ; A_{k} \mid Y_{k}\right)-D_{k}-4 \epsilon H(X)\right.} \\
& \stackrel{\mathrm{f}}{=} \gamma_{n} 2^{-n\left(I\left(W ; U_{k}\right)-H\left(X \mid A_{k}, Y_{k}\right)-4 \epsilon H(X)-\rho\right)} \text {. }
\end{aligned}
$$

$$
\begin{aligned}
& \mathbb{P}\left[\left(\mathbb{X}(\tilde{m}), \mathbb{Y}_{k}, \hat{A}_{k}\right) \in \mathcal{T}_{\epsilon} \mid\left\{\mathbb{X} \neq \mathbb{X}\left(m^{\prime}\right), \forall m^{\prime}<m\right\} \cap\{\mathbb{X}=\mathbb{X}(m)\} \cap \mathcal{S}_{4} \cap\left\{\mathcal{L}_{k}=l\right\}\right] \\
& \stackrel{\text { e.1 }}{=} \mathbb{P}\left[\left(\mathbb{X}(\tilde{m}), \mathbb{Y}_{k}, \mathbb{A}_{k}\right) \in \mathcal{T}_{\epsilon} \mid\{\mathbb{X} \neq \mathbb{X}(\tilde{m})\} \cap\left\{\left(\mathbb{X}(\tilde{m}), \mathbb{Y}_{k}\right) \in \mathcal{T}_{\epsilon}\right\} \cap\left\{\left(\mathbb{X}, \mathbb{Y}_{k}, \mathbb{A}_{k}\right) \in \mathcal{T}_{\epsilon_{1}}\right\}\right] \\
& \stackrel{\text { e.2 }}{\leq}\left(\frac{1}{\mathbb{P}\left[\mathbb{X} \neq \mathbb{X}(\tilde{m}) \mid\left\{\left(\mathbb{X}(\tilde{m}), \mathbb{Y}_{k}\right) \in \mathcal{T}_{\epsilon}\right\} \cap\left\{\left(\mathbb{X}, \mathbb{Y}_{k}, \mathbb{A}_{k}\right) \in \mathcal{T}_{\epsilon_{1}}\right\}\right]}\right) \\
& \left(\frac{\mathbb{P}\left[\left(\mathbb{X}(\tilde{m}), \mathbb{Y}_{k}, \mathbb{A}_{k}\right) \in \mathcal{T}_{\epsilon} \mid\left(\mathbb{Y}_{k}, \mathbb{A}_{k}\right) \in \mathcal{T}_{\epsilon_{1}}\right]}{\mathbb{P}\left[\left(\mathbb{X}(\tilde{m}), \mathbb{Y}_{k}\right) \in \mathcal{T}_{\epsilon} \mid \mathbb{Y}_{k} \in \mathcal{T}_{\epsilon_{1}}\right]}\right) \\
& \stackrel{\text { e. } 3}{\leq}\left(\frac{1}{1-\frac{1}{1-\zeta_{n}} 2^{-n\left(H\left(X \mid Y_{k}\right)-3 \epsilon H(X)\right)}}\right)\left(\frac{2^{-n\left(I\left(X ; Y_{k}, A_{k}\right)-2 \epsilon H(X)\right)}}{2^{-n\left(I\left(X ; Y_{k}\right)+2 \epsilon H(X)\right)}}\right) \\
& \stackrel{\text { e. } 4}{=} \gamma_{n} 2^{-n\left(I\left(X ; A_{k} \mid Y_{k}\right)-4 \epsilon H(X)\right)}
\end{aligned}
$$

\section{REFERENCES}

[1] A. Damnjanovic, J. Montojo, W. Yongbin, J. Tingfang, L. Tao, M. Vajapeyam, Y. Taesang, S. Osok, and D. Malladi, "A survey on 3GPP heterogeneous networks," IEEE Wireless Commun, vol. 18, no. 3, pp. $10-21,2011$.

[2] A. Ghosh, N. Mangalvedhe, R. Ratasuk, B. Mondal, M. Cudak, E. Visotsky, T. A. Thomas, J. G. Andrews, P. Xia, H. S. Jo, H. S. Dhillon, and T. D. Novlan, "Heterogeneous cellular networks: from theory to practice," IEEE Commun. Magazine, vol. 50, no. 6, pp. 54 - 64, 2012.

[3] M. K. Karakayali, G. J. Foschini, and R. A. Valenzuela, "Network coordination for spectrally efficient communications in cellular systems," IEEE Wireless Commun., vol. 13, no. 4, pp. 56-61, 2006.

[4] D. Gesbert, S. Hanly, H. Huang, S. Shamai (Shitz), O. Simeone, and Y. Wei, "Multi-cell MIMO cooperative networks: a new look at interference," IEEE J. Sel. Areas Commun., vol. 28, no. 9, pp. 1380 1408, 2010.

[5] J. G. Andrews, "Interference cancellation for cellular systems: a contemporary overview," IEEE Wireless Commun., vol. 12, no. 2, pp. 19 29, 2005.
[6] V. Chandrasekhar and J. G. Andrews, "Spectrum allocation in tiered cellular networks," IEEE Trans. Commun., vol. 57, no. 10, pp. $3059-$ 3068, 2009.

[7] S. Singh, H. S. Dhillon, and J. A. Andrews, "Offloading in heterogeneous networks: modeling, analysis, and design insights," IEEE Trans. Wireless Commun., vol. (to appear), 2014.

[8] E. Tuncel, "Slepian-Wolf coding over broadcast channels," IEEE Trans. Inform. Theory, vol. 52, no. 4, pp. 1469 -1482, 2006.

[9] Y.-H. Kim, "Capacity of a class of deterministic relay channels," IEEE Trans. Inform. Theory, vol. 54, no. 3, pp. $1328-1329,2008$.

[10] A. Wyner, "On source coding with side-information at the decoder," IEEE Trans. Inform. Theory, vol. 21, no. 3, pp. 294-300, 1975.

[11] T. J. Oechtering, C. Schnurr, I. Bjelakovic, and H. Boche, "Broadcast capacity region of two-phase bidirectional relaying," IEEE Trans. Inform. Theory, vol. 54, no. 1, pp. 454-458, 2008.

[12] G. Kramer and S. Shamai, "Capacity for classes of broadcast channels with receiver side information," in proc. IEEE Inform. Theory Workshop, Lake Tahoe, California, 2007.

[13] A. Wyner, J. Wolf, and F. Willems, "Communicating via a processing 
broadcast satellite," IEEE Trans. Inform. Theory, vol. 48, no. 6, pp. 1243-1249, 2002.

[14] R. Timo, A. Grant, and G. Kramer, "Lossy broadcasting with complementary side information," IEEE Trans. Inform. Theory, vol. 59, no. 1, pp. $104-131,2013$.

[15] T. Cover, "A proof of the data compression theorem of Slepian and Wolf for ergodic sources," IEEE Trans. Inform. Theory, vol. 21, no. 2 , pp. 226-228, 1975.

[16] D. Gunduz, E. Erkip, A. Goldsmith, and H. V. Poor, "Source and channel coding for correlated sources over multiuser channels," IEEE Trans. Inform. Theory, vol. 55, no. 9, pp. 3927-3944, 2009.

[17] J. Nayak, E. Tuncel, and D. Gunduz, "Wyner-Ziv coding over broadcast channels: digital schemes," IEEE Trans. Inform. Theory, vol. 56, no. 4, pp. 1782-1799, 2010.

[18] R. Timo, G. Lecher, L. Ong, and S. Johnson, "Multi-way relay networks: orthogonal uplink, source-channel separation and code design," IEEE Trans. Commun., vol. 61, no. 2, pp. 753 - 768, 2013.

[19] D. Gündüz, E. Erkip, A. Goldsmith, and H. V. Poor, "Reliable joint source-channel cooperative transmission over relay networks," IEEE Trans. Inform. Theory, vol. 59, no. 4, 2013.

[20] D. Blackwell, L. Breiman, and A. J. Thomasian, "Capacity of a class of channels," The Annals of Mathematical Statistics, vol. 30, no. 4, pp. 1229 - 1241, 1959.

[21] A. Lapidoth and E. Telatar, "The compound channel capacity of a class of finite-state channels," IEEE Trans. Inform. Theory, vol. 44, no. 3, pp. 973-983, 1998.

[22] D. Slepian and J. Wolf, "Noiseless coding of correlated information sources," IEEE Trans. Inform. Theory, vol. 19, no. 4, pp. 471-480, 1973.

[23] T. Cover and J. Thomas, Elements of Information Theory. John Wiley and Sons, 2006.

[24] A. Wyner and J. Ziv, "The rate-distortion function for source coding with side information at the decoder," IEEE Trans. Inform. Theory, vol. 22, no. 1, pp. 1-10, 1976.

[25] C. Chen, D.-k. He, and A. Jagmohan, "The equivalence between SlepianWolf coding and channel coding under density evolution," IEEE Trans. Commun., vol. 57, no. 9, pp. 2534-2540, 2009.

[26] K. M. Morshed, I. Land, and G. Lechner, "Lossless compression of uniform binary sources with coded side-information," proc. Australian Commun. Theory Workshop, Sydney, Australia, 2014.

[27] C. Heegard and T. Berger, "Rate distortion when side information may be absent," IEEE Trans. Inform. Theory, vol. 31, no. 6, pp. 727-734, 1985.

[28] R. Timo, T. Chan, and A. Grant, "Rate distortion with side-information at many decoders," IEEE Trans. Inform. Theory, vol. 57, no. 8, pp. 5240-5257, 2011.

[29] A. El Gamal and Y.-H. Kim, Network Information Theory. Cambridge University Press, 2011.

[30] Y.-K. Chia, "On multiterminal source coding with list decoding constraints," in Intl. Symp. Inform. Theory, HI, USA, 2014.

[31] Y.-H. Kim, A. Sutivong, and T. M. Cover, "State amplification," IEEE Trans. Inform. Theory, vol. 54, no. 5, pp. 1850 - 1859, 2008.

[32] R. W. Yeung, Information theory and network coding. Springer, 2008

[33] G. Kramer, "Topics in multi-user information theory," Foundations and Trends in Communications and Information Theory, vol. 4, no. 45, pp. 265-444, 2008.

[34] G. Kramer, "Lecture notes on Multi-User Information Theory," LNT, Technische Universität München, 2012.

[35] A. Wyner, "A theorem on the entropy of certain binary sequences and applications: part I,’ IEEE Trans. Inform. Theory, vol. 19, no. 6, pp. 769$772,1975$.

[36] A. Wyner, "A theorem on the entropy of certain binary sequences and applications: part II," IEEE Trans. Inform. Theory, vol. 19, no. 6, pp. 772-777, 1975.

[37] R. Ahlswede and J. Körner, "Source coding with side information and a converse for degraded broadcast channels," IEEE Trans. Inform. Theory, vol. 21 , no. 6 , pp. $629-637,1975$.

[38] T. Cover, A. E. Gamal and M. Salehi, "Multiple access channels with arbitrarily correlated sources," IEEE Trans. Inform. Theory, vol. 26, no. 6, pp. $648-657,1980$.

[39] O. İşcan, I. Land and G. Lechner, "Repeat-Accumulate Codes for Broadcast Channels with Side Information," proc. Intl. ITG Conf. Systems, Commun. Coding, Hamburg, Germany, Feb., 2015. 